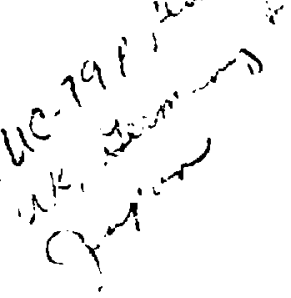

Issued: November 1976

$\hat{i}$

by

H. F. Martz, Jr.

R. A. Waller

Handbook of Bayesian Reliability Estimation Methods

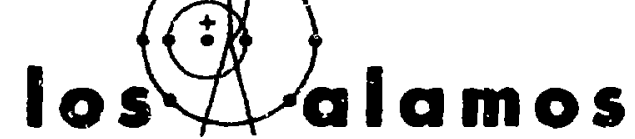

\title{
seientifio laboratory
}

of the University of California

LOS ALAMOS, NEW MEXICO 87545

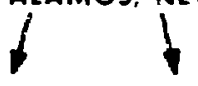

An Affirmative Astion/Equal Opporiunity Employer 
This work supported by the US Energy Research and Development Administration, Division of Reactor Development and Demonstration, Reactor Safety Branch.

Printed in the United States of America. Available from

National Technical Information Service

U.S. Department of Commerce

5285 Port Royal Roed

Springrield, VA 22161

Pice: Printed Copy \$4.50 Mirrofiche $\$ 3.00$

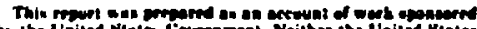

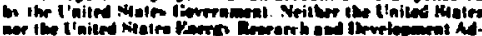

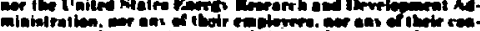

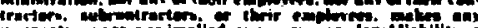

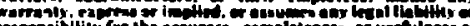

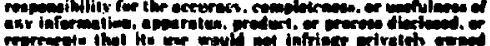

moreme. 
HANDBOOK OF BAYESIAN RELIABILITY ESTIMATION METHODS

by

H. F。 Martz, Jr., and R. A. Waller

\begin{abstract}
Bayesian reliability estimation methods are summarized in a handbook format for convenient use by reliability practitioners. The methods given consider both attribute test data based on a binomial sampling distribution and a beta prior, as well as variables test data from an exponential sampling distribution and a gamma pricr. Classical, Bayes, and empirical Bayes methods are all considered. In addition, the sample test data can arise from either an item-censored life test, either with or without the replacement of failed items as they occur, or from a time-truincated life test with replacement. Real-data examples using nuclear reactor component failure data are used to illustrate each of the methods presented.
\end{abstract}

\title{
1. INTRODUCTION
}

I.A. Notation and Definitions

The results summarized in this handbook reiy heavily on the more complete development provided in Waller and Martz and Martz and Waller. ${ }^{2}$ However, the notation used here may depart from that of the preceding documents in order to provide consistency throughout the handbook. Some general. notation is presented below, while special notation required will accompany the methods to ta presented.

General Notation:

$n$, the number of items on test 
$x$, the observed number of survivors of a test of duration $t$

$t_{i}$, the $i^{\text {th }}$ failure time

$r$, the observed number of failures $(c<r<n$ )

$t_{0}$, the time of cest termination

$\left(t_{1}<t_{2}<\cdots<\tau_{2}\right)$, a sample of $r$ ordered times-tofailure

$\tau=\sum_{i=1}^{r} t_{i}+(n-r) t_{r}$,

the total test time when the testing is terminated at the time of the $r^{\text {th }}$ failure and

failed items are not replaced

(Type II/icem-censored testing without replacemen:)

$T=n x_{r}$, the total test time when the resting is terminated at the time of the $r^{\text {th }}$ failure and failed items are replaced (Type II/item-censored testing with replacement)

$T=n t_{0}$, the total test time when the testing is terminated at time $\delta_{0}$ and failed itens are replaced (Type I/ time-truncated testing with replacement)

$v$, degrees of freedon of the chi-square distribution

$v_{1}$, numerator degrees of freedon of the f distribution; degrees of freedom of the chi-square distribution for lower interval end points

$v_{2}$, denominator degrees of freedom of the f distribution; degrees of freedom of the chi-square distribution for upper interval end points

$x_{a ; v}^{2}, 100(a)$ th percentile of a chi-square distribution with $v$ degrees of freedon $\left[P\left(x^{2}<x_{a ; v}^{2}\right)=0\right]$

$F_{a ; v_{1}}, v_{2}, 100(a)$ th percentage point of an I distribution with $v_{1}$ numerator and $v_{2}$ denominator degrees of freedom $\left[P\left(F>F_{a ; v_{1}, v_{2}}\right)=a\right]$

$R$, reliability; the probability that an item will operate Accessfully for a specifiel length of time when used 
under specified conditions; the probability of failureEree operation for a specified length of time

$R(t)$, reliabijity; the probability that an item will operate successfully for a length of time $t$ when used under specified conditions

PWR , pressurized water reactor

$\lambda$, failure-rate (failures/unit-time)

$n_{0}$, beta priot distribution parameter representing "pseudo sample size"

$x_{0}$, beta prior distribution parameter representing "pseudo number of survivors"

ao gamna priot distribution shape parameter

$B_{0}$, gamina prior distribution scale parameter

$r(x)$, gamma function

$1-\alpha$, confidence that a confidence-interval estimate contains the specified reliability parameter

1-Y , probability that a probability-interval estimate con-

tains the specified reliability parameter

TC1, two-sided confidence interval

LCl, lower one-sided confidence interval

UCl, upper one-sided confidence interval

TBPI, two-sided Bayes probability interval

LBPI, lower one-sided Bayes probability interval

UBPI, upper one-sided bayes probability interval

TEBPI, two-sided empirical Bayes probability interval

LEPI, lower one-sided empirical Bayes probability interval

UEBP 1 , upper one-sided empirical Bayes probability interval

Note: When " 0 " accomparies any of the Bayes probability-interval abbreviations, it implies that the interval estimate is based entirely on the prior distribution; that is, the interval is a projected estimate before actual observed failure data become available. In References 1 and 2, such an estimate is referred to as a "no data estimate." On the other hand, if a Bayes interval estimate is derived 
from the posterior distribution, which includes sample test information from a sample of size $n$, the lotter " $n$ " will accompany the abbreviation. Such estimates are referred to as "data estimates." A "no data estimate" is the result of a prior analysis, while a "data estimate" is the result of a posterior analysis (see Definitions below).

Definitions:

Prior Information - information which exists about a reliability parameter of interest before sample test data become available. The information may be zither subjective or objective.

Attribute Test Data - data in which only the survival/nonsurvival of each item on life test is recorded.

Variables Test Data - data in which the time-to-failure of each item failing during a life test is recorded.

Sample Test Data - objective reliability failure information obtained from a set of items which have been placed on life test. The data may be either attribute or variables data.

Sampling Distribution - the statistical distribution which is assumed for the sanple test data.

Classical Estimation - those estimation techniques which utilize only objective sample test data in computing the estimates.

Bayesian Estimation - an estimation methodology which formally pernits combining two sources of information about a reliabjility parameter of interest. The two sources are prior information and objective sample test data.

Empirical Bayes Estimation - an estimation methodolngy which is Bayesian in nature but with fewer assumptions regarding the prior distribution.

Prior Analysis - reliability estimates derived from the prior distribution before sample test data are available. 
Posterior Analysis - reliability estimates derived from the posterior distribution when sample test data are available for Bayesian analysis.

Prior Distribution - a statistical distribution for the reliability parameter of interest in a Bayesian analysis. This distribution summarizes the prior information about the reliability parameter.

Posterior Distribution - the statistical distribution obtained by combining the prior distribution ard the sampling distribution by means of Bayes theorem.

Confidence Interval - an interval estimator which is said to contain a reliability parameter of interest with a specified confidence.

Probability Interval - an interval estimator which contains the reliability parameter of interest $v$ ith a specified probability.

Point Estimate - a single number which best estimates the reliabjlity parameter of interest as opposed to an interval estimate.

Item-Censored Life test- a life test experiment which is terminated after a prespecified number of failures have been observed.

Tine-Truncated Life test- a life test experiment which is terminated after a prespecified length of test time has elapsed.

Probability Distributions Considered:

Exponential Probability Density Function -

$$
f(t)=\lambda \exp (-\lambda t), t>0 ; \lambda>0
$$

Gamma Prior Probability Density Function -

$$
g(\lambda)=\frac{\lambda^{\alpha_{0}-1} e^{-\lambda / \beta_{0}}}{\beta_{0}^{\alpha_{0}} \Gamma\left(\alpha_{0}\right)}, \lambda>0 ; \alpha_{0}, \beta_{0}>0
$$


Binomial Distribution -

$P(x)=\frac{n !}{(n-x) ! x !} R^{x}(1-R)^{n-x}, x=0,1, \ldots, n ; 0<R<1$.

Beta Prior Probability Density Function -

$$
g(R)=\frac{\Gamma\left(n_{0}\right)}{\Gamma\left(x_{0}\right) \Gamma\left(n_{0}-x_{0}\right)} R^{x_{0}^{-1}}(1-R)^{n_{0}-x_{0}-1}, \begin{aligned}
& 0<R<1 ; \\
& n_{0}>x_{0}>0 .
\end{aligned}
$$

I.B. Handbook Usage

A user of this handbook will ge through a process of elimination to classify his problem of interest into one of the categories contained in Sec. III. A diagram to assist in this elimination process is presented in Figure 1.

For either Bayes or empirical Bayes methods, the information in Sec. II will be helpful in selecting a prior distribution.

\section{I.C. Scope}

The scope of the variables test sata methods presented in this handbook covers both point and interval estimators of reliability and failure rates for the following three general categories of methods: (1) Classical, (2) Bayes, and (3) Empirical Bayes.

The classical estimation of reliability is limited to maximum likelihood estimation for point estimates and confidence intervals based on the chi-square distribution. The only sampling distribution considered is the exponential (constant failure rate) distribution. The sample test data are assumed to arise from either an item-censored or time-truncated life test situation. The item-censored test can be conducted either with or without the replacement of failed items while the time-truncated test assumes that failed items will be replaced as they occur.

The Bayes probability intervals are likewise given in terms of percentiles of the chi-square distribution. As discussed in 


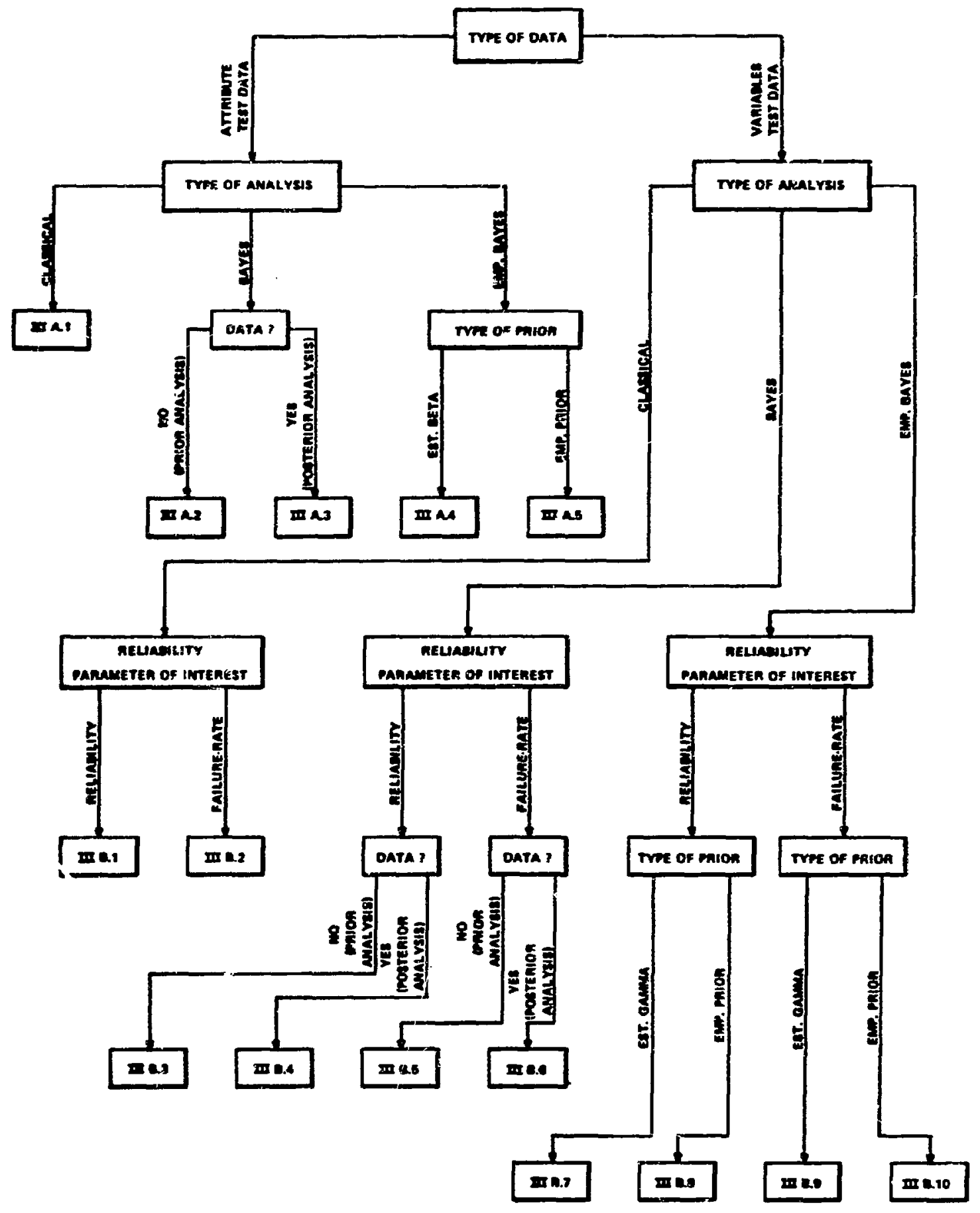

Fig. 1. A Diagram to Assist the Handbook User. 
Waller and Martz, I a gamma prior distribution of the failure rate $\lambda$ is assumed throughout Sec. III.B.

The scope of the attribute test data methods presented in this handbook covers both point and interval estimators of the probability of failure-free operation for a specified length of time (the reliability) for the following three general categories of methods: (1) Classical, (2) Bayes, and (3) Empirical Bayes.

The classical estimation of reirability is limited to maximum likelihood estimation for point estimates and confidence intervals are given in terms of percentage points of the $F$ distribution. For reasons discussed in Martz and Waller, ${ }^{2}$ a beta prior distribution of reliability is assumed throughout Sec. III.A. It is noted that all the interval estimates in Sec. III.A are given in terms of the $F$ distribution. The Bayes interval estimates consider appropriate areas under a beta distribution. Since this distribution is not widely tabulated, equivalent estimates are expressed in terms of the $F$ distribution, which is widely tabulated.

\section{SELECTING A PRIOR DISTRIBUTION}

II.A. General Remarks

Use of the results in this handbook requires that certain parameter values be specified. The purpose of this section is to indicate which parameters must be specified, to motivate the nature of those parameters, and to provide references to some existing procedures which may be helpful in the necessary value specifications. The discussion is divided into two parts. First, Sec. B addresses the selection of beta parameters for priors used in reliability estimation for attribute testing. Second, Sec. C presents a discussion of selecting parameters for gamma priors on failure rates in exponential time-to-failure models. 
II.B. Beta Priors for Reliability Estimation in Attribute

Testing Case

We suppose that $R$, the reliability of an iten being investigated by an attribute testing experiment, is a random variable slich that the prior density of $k$ is given by the beta distribution

$$
g(R)=\frac{\Gamma\left(n_{0}\right)}{\Gamma\left(x_{0}\right) \Gamma\left(n_{0}-x_{0}\right)} R^{x_{0}^{-1}}(1-R)^{n_{0}-x_{0}-1}, 0 \leqslant R \leqslant 1 ; n_{0}>x_{0}>0 .
$$

The experimenter must then select values for the parameters $n_{0}$ and $x_{0}$. For reference, it is convenient to think of $n_{0}$ and $x_{0}$ as pseudo number of trials and pseudo number of successes, respectively.

Waterman, Martz, and Waller ${ }^{3}$ present an extensive set of tables which assist an experimenter with the translation of experiences, judgments, and beliefs into numerical choices for $n_{0}$ and $x_{0}$. Those tables require an experimenter to specify values for either the 5 th percentile and the mean for $R$, or the inean and 95 th percentiie for $R$.

\section{Ii.C. Selection of paralliter lalues for a Gama prior on}

\section{Failure Rate}

If we assume that the time-to-failure for an iten is exponentially distributed with failure rate $\lambda$, the conjugate prior distribution for $\lambda$ is the gamma density given by

$$
g(\lambda)=\frac{\lambda^{\alpha_{0}-1} e^{-\lambda / \beta_{0}}}{\beta_{0}^{\alpha_{0}} \Gamma\left(\alpha_{0}\right)}, \lambda>0 ; \alpha_{0}, \beta_{0}>0 .
$$

To use the Bayesian methods presented in this handbook for exponential failure data, an experimenter must specify values for the parameters $\alpha_{0}$ and $\beta_{0}$. We may note that the prior expected value of $\lambda$ ic $E(\lambda)=\alpha_{0} \beta_{0}$ and the prior variance of $\lambda$ is $V(\lambda)=\alpha_{0} \beta_{0}^{2}$. 
Thus, one method of specifying values for $\alpha_{0}$ and $\beta_{0}$ is to subjectively (using ali available experiences, beliefs, etc.) select values for $E(\lambda)$ and $V(\lambda)$ and specify $\alpha_{0}=E^{2}(\lambda) / V(\lambda)$ and $\beta_{0}$ $=V(\lambda) / E(\lambda)$. That method is presented along with tables for specifying values for one of the pairs of percentiles, $(5,50)$, $(5,95)$, or $(50,95)$ in Martz and Horita ${ }^{4}$. If an experimenter wants to use reliability experience in specifying values for $\alpha_{0}$ and $\beta_{0}$, then Waller, Mar: $z$, Horita, and Waterman ${ }^{5}$ provide tables and graphs to assist in translating values for two re1 iability percentiles, say $(5,50),(5,95)$, or $(50,95)$, into values for $\alpha_{0}$ and $\beta_{0}$ in the gamma prior for $\lambda$.

III. POINT AND INTERVAL ESTIMATION METHODS FOR RELIABILITY AND FAILURE-RATES

III.A. Attribute Test Data

For the results in this section, we suppose that $n$ items are placed on life test for a specified length of time and that $x$ survivors are observed. The number of survivors follows a binomial distribution isee Martz and Waller. ${ }^{2}$ It is desired to estimate the probability of surviving the test (the reliability or the probability of failure-free operation). The interval-estimation equations are presented in terms of percentage points of the $F$ distribution which are given in the table in Appendix $C$. 
III.A.1. Attribute test data: classical procedures.

a. Point estimator of R:

$$
\hat{R}=x / n . \quad(L A-6126, E q \cdot 2)
$$

b. Two-sided confidence-intervaj, estimator:

$$
100(1-\alpha) \div \operatorname{TCI}: \frac{x}{x+(n-x+1) F_{\alpha / 2 ; 2 n-2 x+2,2 x}}
$$

$(\mathrm{LA}-6126, \mathrm{Eq} \cdot 3)$

$$
\leqslant R \leqslant \frac{(x+1) F_{\alpha / 2 ; 2 x+2,2 n-2 x}}{(n-x)+(x+1) F_{\alpha / 2 ; 2 x+2,2 n-2 x)}}
$$

c. Lower one-sided confidence-interval estimator:

$$
100(1-\alpha) \& L C I: R>\frac{x}{x+(n-x+1) F_{\alpha ; 2 n-2 x+2,2 x}} .
$$

$(\mathrm{LA}-6126, \mathrm{Eq} \cdot \mathrm{4})$

d. Reinarks :

The results in $a, b$, and $c$ above assume that the $n$ items on test survive independently, each with the same probability $R$.

e. Exampie:

Consider Data Set 1 in Appendix A. Fur the Indian Point 1 reactor, the estimated pump reliability for 1972 is $\hat{R}=49 / 50=0.98$. A $95 \%$ two-sided confidenceinterval estimate of this reliability is

$$
\frac{49}{49+2(2.94)} \leqslant \mathrm{R}<\frac{50(39.49)}{1+50(39.49)} \text { or } 0.89 \leqslant R \leqslant 1.00 \text {. }
$$


f. General references:

(i) A. H. Bowker and G. J. Lieberman, Engineering Statistics (Prentice Hall, New York, 1972) 2nd Ed.

(ii) W. J. Dixon and F. J. Massey, Jr., Introduction to Statistical Analysis (McGraw-Hill, New York, 1969) 3rd Ed (see F tables, pp. 470-485). 
III.A.2. Attribute test data: Bayes with known beta prior prior analysis.

a. Point estimator of $\mathrm{R}$ :

$\tilde{R}_{0}=x_{0} / n_{0}$

$(\mathrm{LA}-6126, \mathrm{Eq} \cdot 7)$

b. Two-sided probability-interval estimator:

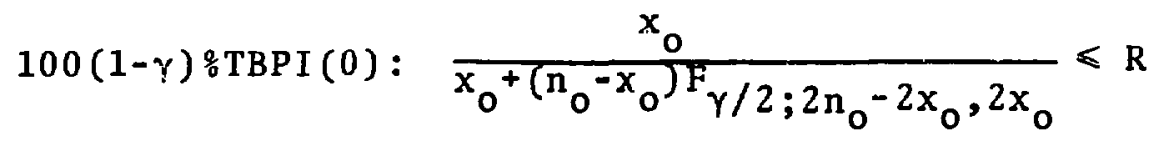

$(L A-6126, E q .8)$

$$
\leqslant \frac{x_{0} F_{Y / 2} ; 2 x_{0}, 2 n_{0}-2 x_{0}}{n_{0}-x_{0}+x_{0} F_{\gamma / 2 ; 2 x_{0}, 2 n_{0}-2 x_{0}}} \text {. }
$$

c. Lower one-sided probability-interval estimator:

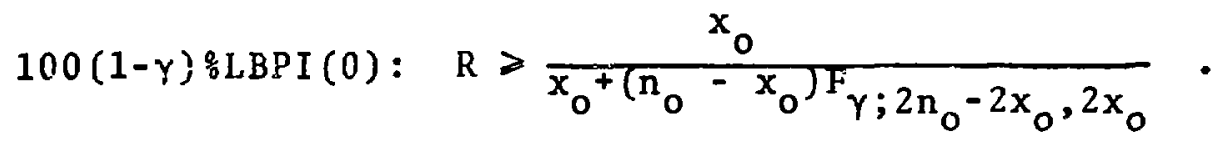

$$
(\mathrm{LA}-6126, \mathrm{Eq} \cdot 9)
$$

d. Remarks :

No current test data is incorporated in the above estimators. They depend only on the prior parameter values $n_{0}$ and $x_{0}$. For guidance in determining $n_{0}$ and $x_{0}=\operatorname{see} \operatorname{Sec}$. II.B. 1 .

e. Example:

Consider Data Set 1 in Appendix A. Suppose that the PWR annual prior mean pump reliability is believed to be 0.95 (prior to the 1972 data). Further, suppose that it is believed that there is only a 5\% chance that the annual pump reliability is below 0.70. From Reference (ii) below, this prior belief is consistent with a 
beta prior distribution with $x_{0}=2.43675$ and $n_{0}$ $=2.56500$. Prior to the 1972 data, the pump reliability is estimated to be $\hat{R}_{0}=2.43675 / 2.56500=0.95$ and a two-sided $95 \%$ probability-interval estimate of this reliability is

$$
\begin{aligned}
& \frac{2.43675}{2.43675+(2.56500-2.43675)(10.01)}<\mathrm{R}< \\
& \frac{2.43675(921.8)}{2.56500-2.43675+2.43675(921.8)},
\end{aligned}
$$

or

$$
0.65 \leqslant R<1.00
$$

Note: Since $2 \mathrm{n}_{0}-2 \mathrm{x}_{0}=0.2565$ and $2 \mathrm{x}_{0}=4.8735$, we approximate $\mathrm{F}_{0.025 ; 0.2565,4.8735 \approx \mathrm{F}_{0.025 ; 1,5}=10.01}$ Similarly, $F_{0.025 ; 4.8735,0.2565} \approx F_{0.025 ; 5,1}=921.8$ Thus, the above interval is only an approximate $95 \%$ probability-interval estimate.

f. General references:

(i) N. R. Mann, R. E. Schafer, and N. D. Singpurwalla, Methods for Statistical Analysis of Reliability and Life Data (John Wiley and Sons, New York, 1974).

(ii) M. S. Waterman, H. F. Martz, and R. A. Waller, "Fitting Beta Prior Distributions in Bayesian Reliability Analysis," Los Alamos Scientific Laboratory report LA-6395-MS (June 1976). 
III.A.3. Attribute test data: Beta with known beta prior posterior analysis.

a. Point estimator of $\mathrm{k}$ :

$$
\tilde{R}=\frac{x+x_{0}}{n+n_{0}}
$$

b. Two-sided probability-interval estimator:

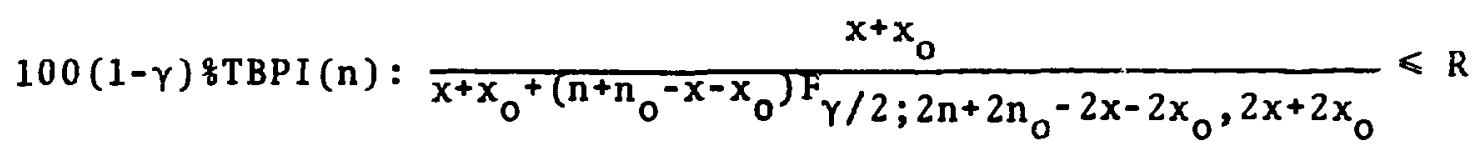

$$
<\frac{\left(x+x_{0}\right) F_{\gamma / 2} ; 2 x+2 x_{0}, 2 n+2 n_{0}-2 x-2 x_{0}}{n+n_{0}-x-x_{0}+\left(x+x_{0}\right) F_{\gamma / 2 ; 2 x+2 x_{0}, 2 n+2 n_{0}-2 x-2 x_{0}}}
$$

c. Lower one-sided probability-interval estimator:

$$
100(1-p) \% \operatorname{LBPI}(\mathrm{n}): \mathrm{R}>
$$

$$
\frac{x+x_{0}}{x+x_{0}+\left(n+n_{0}-x-x_{0}\right) F_{\gamma} ; 2 n+2 n_{0}-2 x-2 x_{0}, 2 x+2 x_{0}}
$$

$(\mathrm{LA}-6126, \mathrm{Eq} \cdot 12)$

d. Remarks :

The results in $a, b$, and $c$ above assume that both the sample data, $n$ and $x$, and the priot values, $n_{0}$ and $x_{0}$, are available. The $n$ test items are assumed to survive independently with probability $R$.

c. Example:

Consider Data Set 1 in Appendix $A$. Suppose that the PWR annual prior mean pump reliability is believed to be 0.95 . Further, suppose that it is believed that 
there is only a $5 \%$ chance that the annual punp reliability is below 0.70. From Reference (ii) below, this prior belief is consistent with a beta prior distribution with $x_{0}=2.43675$ and $n_{0}=2.56500$. In 1972, six pump failures occurred out of 400 pumps. Thus $x=394$ and $n=400$. The updated (posterior) estimated annuai pump reliability is $R=(394+2.43675) /(400+2.56500)$ $=0.98$. A two-sided $95 \%$ probability-interval estimate of this reliability is

$$
\begin{aligned}
& \frac{394+2.43675}{394+2.43675+(400+2.56500-394-2.43675)(1.95)} \leqslant R \\
& \leqslant \frac{(394+2.43675)(2.73)}{400+2.56500-394-2.43675+(394+2.43675)(2.73)},
\end{aligned}
$$

or

$$
0.97 \leqslant R \leqslant 0.99 .
$$

Note: Since $\left(2 n+2 n_{0}-2 x-2 x_{0}\right)=12.2565$ and $\left(2 x+2 x_{0}\right)$ $=792.8735$, we approximate $F_{0} 025 ; 12.2565,792.8735$

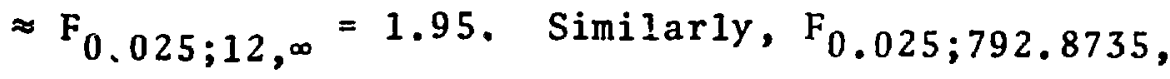
$2.2565 \approx F_{0.025 ; \infty, 12}=2.73$. Thus, the above interval is only an approximate 958 probability-interval estimate.

f. General references:

(i) N. R. Mann, R. E. Schafer, and N. D. Singpurwalla, Methods for Statistical Analysis of Reliability and Life Data (John Wiley and Sons, New York 1974).

(ii) M. S. Waterman, H. F. Martz, and R. A. Waller, "Fitting Beta Prior Distributions in Bayesian Reliability Analysis," Los Alamos Scientific Laboratory report LA-6395-MS (June, 1976). 
III.A.4. Attribute test data: Empirical Bayes--estimated beta parameters.

a. Point estimators of $R$ :

$$
\begin{array}{rlrl}
\tilde{R}_{E} & =\hat{x}_{0} / \hat{n}_{0} \text { (prior analysis), } & \text { (analogous to } \\
\text { LA-6126, Eq. 7) }
\end{array}
$$

where

$$
\hat{x}_{0}=\hat{n}_{0}\left(\Sigma \hat{R}_{2}\right) / N \text {, }
$$

and

$$
\hat{n}_{0}=\frac{N^{2}\left(\Sigma \hat{R}_{j}-\Sigma \hat{R}_{j}^{2}\right)}{N\left(N \Sigma \hat{R}_{j}^{2}-K \Sigma \hat{R}_{j}\right)-(N-K)\left(\Sigma \hat{R}_{j}\right)^{2}},
$$

$(\mathrm{LA}-6126, \mathrm{Eq} \cdot 13)$

if the result is positive, otherwise

$$
\hat{n}_{0}=\left(\frac{N-1}{N}\right) \frac{N \Sigma \hat{R}_{j}-\left(\Sigma \hat{R}_{j}\right)^{2}}{N \Sigma \hat{R}_{j}^{2}-\left(\Sigma \hat{R}_{j}\right)^{2}}-1 \text {. }
$$

b. Two-sided probavility-interval estimator:

$100(1-\gamma) \operatorname{TEBPI}(0): \frac{\hat{x}_{0}}{\hat{x}_{0}+\left(\hat{n}_{0}-\hat{x}_{0}\right) F_{\gamma / 2 ; 2 \hat{n}_{0}-2 \hat{x}_{0}, 2 \hat{x}_{0}}}<R$

(prior analysis)

(analogous to LA-6126, Eq. 8)

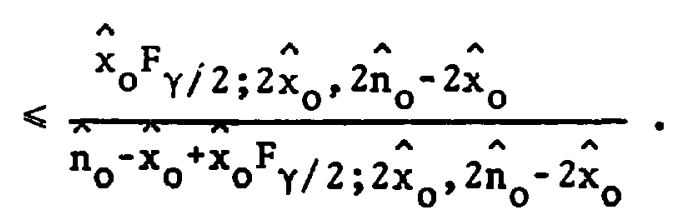




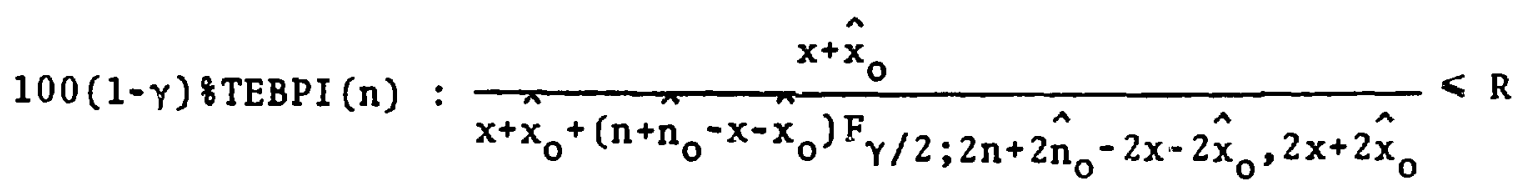

(analogous to

LA-6126, Eq. 11)

$$
<\frac{\left(x+\hat{x}_{0}\right) F_{\gamma / 2} ; 2 x+2 \hat{x}_{0}, 2 n+2 \hat{n}_{0}-2 x-2 \hat{x}_{0}}{n+\hat{n}_{0}-x-\hat{x}_{0}+\left(x+\hat{x}_{0}\right) F_{\gamma / 2} ; 2 x+2 \hat{x}_{0}, 2 n+2 \hat{n}_{0}-2 x-2 \hat{x}_{0}} \text {. }
$$

(posterior analysis)

c. Lower one-sided probability-interval estimators:

$100(1-\gamma) \& \operatorname{LEBPI}(0): R \geqslant \frac{\hat{x}_{0}}{\hat{x}_{0}+\left(\hat{n}_{0}-\hat{x}_{0}\right) F_{\gamma ; 2 \hat{n}_{0}-2 \hat{x}_{0}, 2 \hat{x}_{0}}}$,

(prior analysis)

(analogous to

LA-6126, Eq. 9)

$100(1-\gamma) \& \operatorname{LEBPI}(n): R>\frac{x+\hat{x}_{0}}{x+\hat{x}_{0}+\left(n+\hat{n}_{0}-x-\hat{x}_{0}\right) F_{Y} ; 2 n+2 \hat{n}_{0}-2 x-2 \hat{x}_{0}, 2 x+2 \hat{x}_{0}}$.

(posterior analysis)

(analogous to

LA-6126, Eq. 12)

d. Remarks :

The results in $a, b$, and $c$ assume a beta prior model with unknown parameters, $n_{0}$ and $x_{0}$. These parameters are estimated from past test data in which, for a series of $\mathrm{N}$ independent past life tests on similar test units, $x_{j}$ survivors have been observed in a sample of sice $n_{j}$ in the $j^{\text {th }}$ test $(j=1,2, \ldots, N)$. It is noted that all 
life tests must be of approximately the same duration. In $a$, all summations range from $j=1$ to $j=N, R_{j}=x_{j} / n_{j}$, and $K=\Sigma\left(1 / n_{j}\right)$.

e. Example:

Consider Data Set 1 in Appendix A. We consider each of the eight PWRs ${ }_{\lambda}$ isted to be an independent life test experiment. Now $\Sigma \hat{R}_{j}=7.88, \Sigma \hat{R}_{j}^{2}=7.7648\left(\Sigma \hat{R}_{j}\right)^{2}=62.0944$, $\mathrm{K}=0.16$ from which

$$
\hat{n}_{0}=\frac{64(7.88-7.7648)}{8[8(7.7648)-0.16(7.88)]-(8-0.16) 62.0944}=181.15,
$$

and

$$
\hat{x}_{0}=\frac{181.15(7.88)}{8}=178.43
$$

Performing a prior analysis on the data in Data Set 1 , the 1972 pump reliability is estimated to be $R_{E}=178.43 / 181.15$ $=0.98$ and a two-sided $95 \%$ probability-interval estimate is

$\frac{178.43}{178.43+(181.15-178.43)(2.57)} \leqslant \mathrm{R}<\frac{178.43(6.02)}{181.15-178.43+178.43(6.02)}$,

or

$$
0.96 \leqslant R \leqslant 1.00
$$

Note: $F_{0.025 ; 5.44,3,56.86} \approx F_{0.025 ; 5, \infty}=2.57$ and $\mathrm{F}_{0.025 ; 356.86,5.44} \approx \mathrm{F}_{0.025 ; \infty, 5}=6.02$. Thus the above interval is only an approximate $95 \%$ probability-interval estimate. 
III.A.5. Attribute test data: empirica1 Bayes -- estimated (empirical) prior

a. Point estimators of R:

$$
\begin{aligned}
\tilde{R}_{E} & =\sum_{j=1}^{N} \hat{R}_{j} / N \quad \text { (prior analysis) } \\
& =\frac{\sum_{j=1}^{N} \hat{R}_{j}^{x+1}\left(1-\hat{R}_{j}\right)^{n-x}}{\sum_{j=1}^{N} \hat{R}_{j}^{x}\left(1-\hat{R}_{j}\right)^{n-x}} \text { (posterior analysis) }
\end{aligned}
$$

b. Two-sided probability-interval estimators: (see LA6126, Eq. 20, except that the empirical prior distribution is used instead of the empirical posterior distribution)

(prior analysis)

(see LA-6126, Eq. 20)

(posterior analysis)

c. Lower one-sided probability-interval estimators: (see LA-6126, Eq. 21 except that the empirical prior distribution is used instead of the empirical posterior distribution)

(prior ana1ysis)

(see LA-6126, Eq. 21)

(posterior analysis)

d. Remarks :

The results in $a, b$, and $c$ do not require an assumption regarding a type of prior model, such as a beta distribution. The complete priur distribution is empir. ically estimated from past test data in which, for a series of $N$ independent past 1 ife tests on similar test units, $x_{i}$ survivors have been observed in a sample of size $n_{i}$ in the $i^{\text {th }}$ test $(i=1,2, \ldots, N)$. It is noted 
that all 1 ife tests must be of approximately the same duration.

The interval estimates should be used with caution. The true coverage probability is likely to be less than that desired unless the number of past data sets $\hat{R}_{1}, \ldots, \hat{R}_{N}$ is greater than 25 .

e. Example:

Consider Data Set 1 in Appendix A. Suppose we de. sire to estimate the 1972 pump reliability of the Indian Point 1 PWR. For Indian Point 1 , we have $n=50$ and $x=49$. Thus

$$
\tilde{R}_{E}=\frac{4(1.00)^{50}(0)^{1}+3(0.98)^{50}(0.02)^{1}+(0.94)^{50}(0.02)^{1}}{4(1.00)^{49}(0)^{1}+3(0.98)^{49}(0.02)^{1}+(0.94)^{49}(0.02)^{1}}=0.978
$$

f. General references:

(i) G. H. Lemon, "An Empirical Bayes Approach to Reliability," IEEE Trans. Re1. R-21, 155-158 (August 1972).

(ii) H. F. Martz, Jr., "Pooling Life-Test Data by Means of the Empirical Bayes Method, "IEEE Trans. Rel. R-24, 27-30 (April 1975). 
III.B. Variables Test Data-Exponential Model

The results in this section assume that the time-to-failure of an item is distributed as an exponential variable. For a general discussion of the exponential distribution, see Sec. II.A of Waller and Martz, ${ }^{1}$ or the references 1 isted with each subsection below. The test data are assumed to arise from either an item-censored or time-truncated experiment. The itemcensored experiment can be conducted either with or without the replacemant of failed items as they occur. The time-truncated experiment assumes that failed items will be replaced as they occur. In addition, for the classical estimators, time-truncated testing without replacement is also considered. It is noted here that if the data arise from field use of an item, the situation will normally be with replacement of failed items and often will be a time-truncated experiment. For the exponential model considered here, the reliability is $R(t)=\exp (-\lambda t)$. Formulas are given in this section for estimating both $R(t)$ and the failure rate $\lambda$. As mentioned earlier, for the Bayes estimators a gamma prior distribution of $\lambda$ is assumed. 
III.B.I. Variables test data: classical procedure--reliability estimation

a. Point estimator of $R(t)$ :

$\hat{R}(t)=\exp (-r t / T)$,

$(\mathrm{LA}-6003, \mathrm{Eq} \cdot \mathrm{8})$

where $T$ is the total test time as defined in Sec. I.A, and $r$ is the observed number of failures.

b. Two-sided confidence-interval estimator:

$$
\begin{aligned}
100(1-\alpha) \div \text { TCI: } & \exp \left[-t x^{2}{ }_{1-\alpha / 2 ; 2 \nu_{1}} /(2 \mathrm{~T})\right]<R(t) \\
\leqslant & \exp \left[-t \chi^{2}{ }_{\alpha / 2 ; 2 \nu_{2}} /(2 \mathrm{~T})\right] \cdot(\mathrm{L}-\mathrm{A}-6003, \text { Eq. 9) }
\end{aligned}
$$

c. Lower one-sided confidence-interval estimator:

$100(1-\alpha) \% L C I: \quad R(t) \geqslant \exp \left[-t x_{1-\alpha ; 2 \nu_{1}} /(2 T)\right]$.

d. Remarks :

The value assigned to $\nu_{1}$ depends upon the type of life test experiment and is given by

$$
\begin{aligned}
v_{1} & =r & & \text { (item-censored life test) } \\
& =r+1 & & \text { (time-truncated 1ife test) }
\end{aligned}
$$

while $v_{2}=r$ regardless of the type of 1 ife test experiment. It is further noted that the confidence intervals fin the case of time-truncated 1 ife testing have only approximately $100(1-\alpha) \%$ confidence associated with them. For exact-interval estimates see Ref. (iii) below. Also, for the case of time-truncated testing without replacement,

$T=\sum_{i=1}^{r} t_{i}+(n-r) t_{0}$ 
e. Examp1e:

Consider Data Set 1 in Appendix A. The test data in Data Set 1 can be considered to have been obtained from a time-truncated experiment with replacement in which $n=400, r=6$, and $T=3504000$. The punp reliability in PWRs for 1972 is estimated to be $\hat{R}(8760)=\exp \left[\begin{array}{lll}-6(8760) / 3 & 504 & 000\end{array}\right]=0.985$.

An approximate $95 \%$ two-sided confidence-interval estimate of this reliability is

$$
\begin{array}{r}
\exp [-8760(26.119) / 7008000] \leqslant R(8760) \\
\leqslant \exp [-8760(4.404) / 7 \quad 008000],
\end{array}
$$

or

$$
0.97<R(8760)<0.99 \text {. }
$$

f. General references:

(i) N. R. Mann, R. E. Schafer, and N. D.

Singpurwalla, Methods for Statistical Analysis of Reliability and Life Data (John Wiley and Sons, New York, 1974).

(ii) I. Miller and J. E. Fruend, Probability and Statistics for Engineers (Frentice-Ha11, New Jersey, 1965).

(iii) B. A. Kozlov and I. A. Ushakov, Reliability Handbook (Ed. by L. H. Koopmans and J. Rosenblatt) (Holt, Rinehart, and Winston, New York, 1970). 
III.B.2. Variables test data: classical procedure -- failurerate estimation

a. Point estimator of $\lambda$ :

$\hat{\lambda}=\mathbf{r} / \mathrm{T}$.

b. Two-sided confidence-interval estimator:

$100(1-\alpha) \% \mathrm{TCI}: \quad \chi_{\alpha / 2 ; 2 \nu_{2}}^{2} /(2 \mathrm{~T})<\lambda<\chi_{1-\alpha / 2 ; 2 \nu_{1}}^{2} /(2 \mathrm{~T})$.

c. Upper one-sided confidence-interval estimator:

$100(1-\alpha) \% \mathrm{UCI}: \quad \lambda \leqslant \chi^{2}{ }_{1-\alpha ; 2 \nu_{1}} /(2 \mathrm{~T})$

d. Remarks:

The value assigned to $v_{1}$ depends on the type of experiment and is given by

$$
\begin{aligned}
\nu_{1} & =r & & \text { (item-censored } 1 \text { ife test) } \\
& =r+1 & & \text { (time-truncated } 1 \text { ife test) }
\end{aligned}
$$

while $\nu_{2}=r$ regardless of the type of 1 ife test experiment. It is further noted that the confidence intervals for the case of time-truncated 1 ife testing have only approximately $100(1-\alpha) \%$ confidence associated with then. For exact-interval estimates see Ref. (iii) below. Also, for the case of time-truncated testing without replacement,

$$
T=\sum_{i=1}^{r} t_{i}+(n-r) t_{0} .
$$


e. Example:

Consjder Data Set 1 in Appendix A. The variables test data in Data Set 1 is considered to have been obtained from a time-truncated experiment with replacement in which $n=400, r=6$, and $T=3504000$. Based on the 1972 data for the eight PWRs, the pump failurerate associated with the failure to run normal mode is estimated to be

$$
\hat{\lambda}=6 / 3504000=1.7 \times 10^{-6} \mathrm{f} / \mathrm{h} .
$$

An approximate 95\% two-sided confidence-interval estimate of this failure rate is

$$
(4.404) / 7008000<\lambda \leqslant(26.119) / 7008000 \text {, }
$$

or

$$
6.3 \times 10^{-7} \mathrm{f} / \mathrm{h}<\lambda<3.7 \times 10^{-6} \mathrm{f} / \mathrm{h} .
$$

f. General references:

(i) N. R. Mann, R. E. Schafer, and N. D.

Singpurwalla, Methods for Statistical Analysis of Reliability and Life Data (John Wiley and Sons, New York, 1974).

(ii) I. Miller and J. E. Freund, Probability and Statistics for Engineers (Prentice-Ha11, New Jersey, 1965).

(iii) B. A. Kozlov and I. A, Ushakov, Reliability Handbook (Ed. by L. H. Koopmans and J. Rosenblatt) (Holt, Rinehart, and Winston, New York, 1970). 
III.B.3. Variables test data: Bayes with known gamma priorreliability estimation -- prior analysis

a. Point estimator of $R(t)$ :

$$
\tilde{R}_{0}(t)=\left(1+\beta_{0} t\right)^{-\alpha_{0}} . \quad(L A-6003, E q \cdot 17)
$$

b. Two-sided probability-interval estimator:

$$
\begin{aligned}
& 100(1-\lambda) \div \operatorname{TBPI}(0): \exp \left[-\beta_{0} t \chi^{2}{ }_{\left.1-\gamma / 2 ; 2 \alpha_{0} / 2\right]<R(t)}\right.
\end{aligned}
$$

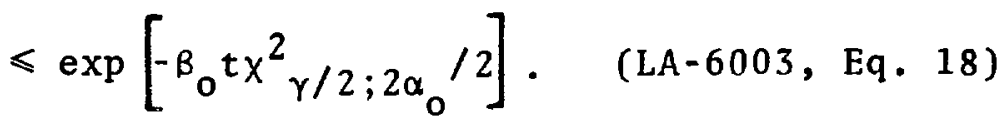

c. Lower one-sided probability-interval estimator:

$$
100(1-\gamma) \div \operatorname{LBPI}(0): \quad R(t) \geqslant \exp \left[-\beta_{0} t x^{2}{ }_{1-\gamma ; 2 \alpha_{0}} / 2\right] \text {. }
$$

d. Remarks:

No current test data is incorporated in the above estimators. They depend only upon the prior parameter values, $\alpha_{0}$ and $\beta_{0}$. For guidance in determining $\alpha_{0}$ and $\beta_{0}$ see Sec. II.C.

e. Example:

Prior to obtaining the data in Data Set 1 , suppose we desire a prior analysis of the annual pump reliability in PWRs. From past experience suppose that we subjectively assign the values 0.50 and $6.0 \times 10^{-6} \mathrm{f} / \mathrm{h}$ to $\alpha_{0}$, and $B_{0}$, respectively. The prior 1972 pump reliability is estimated to be

$$
\tilde{R}_{0}(8760)=\left[1+6.0 \times 10^{-6}(8760)\right]^{-0.50}=0.97 \text {, }
$$

and a two-sided $95 \%$ probability-interval estimate of this reliability is

$$
\left.r a n \times 10^{-6}(8760)(5.024) / 2\right] \leqslant R(8760)
$$




$$
<\exp \left[-6.0 \times 10^{-6}(8760)(0.001) / 2\right]
$$

or

$$
0.88<R(8760)<1.00 \text {. }
$$

f. General reference:

(i) N. R. Mann, R. E. Schafer, and N. D.

Singpurwa1la, Methods for Statistical Analysis of Reliability and Life Data (John Wiley and Sons, New York, 1974 ). 
III.B.4. Variables tese data: Bayes with known gamna prior. reliability estimation - posterior analysis

a. Point estimator of $R(t)$ :

$$
\vec{R}_{r}(t)=\left(\frac{a_{0}^{T+1}}{a_{0} T+0_{0} t+1}\right)^{r+a_{0}} \text {. (1.A-6003,1:q.19) }
$$

b. Two-sided probability-interval estimator:

$$
\begin{aligned}
& 100(1-\gamma) \leqslant \operatorname{tBp} 1(x): \exp \left[-B_{0} t x^{2} 1-\gamma / 2 ; 2 r+2 a_{0} /\left(2 g_{0} T+2\right)\right] \\
& <R(t)<\exp \left[-B_{0} t x^{2} 1-\gamma / 2 ; 2 r+2 a_{0} /\left(2 B_{0} T+2\right)\right] .
\end{aligned}
$$

(Reduces to Fq. 20 of $L A-6003$ when $I=n$.)

c. Lower one-sided probability-interval estimator:

$100(1-\gamma): \operatorname{LBPI}(r): R(t)>\exp \left[-\theta_{0} t^{2}{ }_{\left.1-\gamma ; 2 r+2 \sigma_{0} /\left(2 \theta_{0} T+2\right)\right]}\right.$.

d. Remarks:

$T$ in the above expressions is the total test $t$ ime which is defined in Sec. I.A. The above estimators are appropriate for item-censored 1 ife testing, either with or without replacement, and for time-truncated testing with replacement.

e. Example:

Consider Data Set 1 in Appendix A. Suppose we desire a posterior analysis of the annual pump reliability of the PHRs based on the 1972 failure data reported in Data Set 1. From past experience suppose that we assign the values 0.50 and $6.0 \times 10^{-6} \mathrm{f} / \mathrm{h}$ to $\alpha_{0}$ and $B_{0}$, respectively. In 1972 , six pump failures occurred during a total of approximately 3504000 opr rating 
for the eight PWRs. The testing situation is considered to be time-truncated 1 ife testing with replacement. Thus, $r=6$ and $T=400(8760)=3504000$. The posterior estimated annual pump reliability is

$$
\begin{aligned}
\tilde{R}_{6}(8760) & \left.=\left[\frac{6.0 \times 10^{-6}(3504000)+1}{6.0 \times 10^{-6}(3504000)+6.0 \times 10^{-6}(8760)+1}\right]\right]^{6}+0.5 \\
& =0.98,
\end{aligned}
$$

and a two-sided $95 \%$ probability-interval estimate of the reliability is

$$
\begin{aligned}
& \exp \left\{-\left(6.0 \times 10^{-6}\right)(8760)(24.736) /\left[(2)\left(6.0 \times 10^{-6}\right)(3504000)+2\right]\right\} \\
& <R(8760)<\exp \left\{-\left(6.0 \times 10^{-6}\right)(8760)(5.009) /\right. \\
& \text { or } \left.\quad\left[(2)\left(6.0 \times 10^{-6}\right)(3504000)+2\right]\right\}, \\
& 0.97 \leqslant R(8760)<0.99 .
\end{aligned}
$$

f. General reference:

(i) N. R. Mann, R. E. Schafer, and N. D.

Singpurwalla, Methods for Statistical Analysis of Reliability and Life Data (John Wiley and Sons, New York, 1974). 
III.B.5. Variables test data: Bayes with known gamma prior failure-rate estimation - - prior analysis

a. Point estimator of $\lambda$ :

$$
\tilde{\lambda}_{0}=\alpha_{0} \beta_{0} \text {. }
$$

b. Two-sided probability-interval estimator:

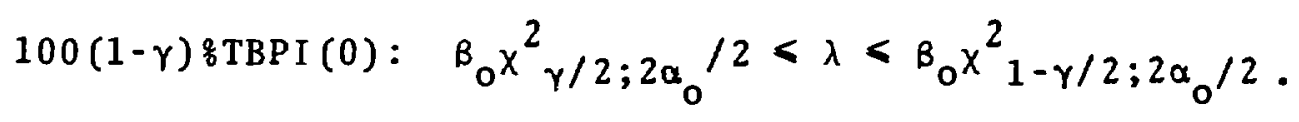

c. Upper one-sided probability-interval estimator:

$$
100(1-\gamma) \div \text { UBPI }(0): \lambda<\beta_{0} \chi^{2}{ }_{1-\gamma ; 2 \alpha_{0}} / 2 \text {. }
$$

d. Remarks:

No current test data are incorporated in the above estimators. They depend only on the prior parameter values $\alpha_{0}$ and $\beta_{0}$. For guidance in determining $\alpha_{0}$ and $B_{0}$, see Sec. II.C.

e. Example:

Prior to obtaining the data in Data Set 1 (Appendix A), suppose we desire a prior analysis of the pump failure rate in PWRs (according to the failure to run normal mode). From past experience suppose that we assign the values 0.50 and $6.0 \times 10^{-6} \mathrm{f} / \mathrm{h}$ to $\alpha_{0}$ and $\beta_{0}$, respectively. The pump failure rate is estimated to be $\tilde{\lambda}_{0}=0.50\left(6.0 \times 10^{-6} \mathrm{f} / \mathrm{h}\right)=3.0 \times 10^{-6} \mathrm{f} / \mathrm{h}$ and an upper 95\% probability-interval estimate of the failure rate is

$$
\lambda<\left(6.0 \times 10^{-6}\right)(3.841) / 2=11.5 \times 10^{-6} \mathrm{f} / \mathrm{h} .
$$

f. General reference:

(i) N. R. Mann, R. E. Schafer, and N. D.

Singpurwalla, Methods for Statistical Analysis of Reliability and Life Data (John Wiley and Sons, New York, 1974) 
III.B.6. Variables test data: Bayes with known gamma prior failure - rate estimation -- posterior analysis

a. Point estimator of $\lambda$ :

$$
\tilde{\lambda}_{T}=\frac{B_{0}\left(r+a_{0}\right)}{B_{0} T+I} .
$$

b. Two-sided probability-interval estimator:

$$
\begin{aligned}
& 100(1-\gamma) \operatorname{TBPI}(r):{ }_{0} \chi^{2} \gamma / 2 ; 2 r+2 \alpha_{0} /\left(2 \beta_{0} T+2\right)<\lambda \\
& <\beta_{0} x^{2}{ }_{1-\gamma / 2 ; 2 r+2 \alpha_{0}} /\left(2 \beta_{0} T+2\right) \text {. }
\end{aligned}
$$

c. Upper one-sided probability-interval estimator:

$$
100(1-\gamma) q U B P I(r): \quad \lambda<\beta_{0} X^{2} 1-Y ; 2 r+2 \alpha_{0} /\left(2 \beta_{0} T+2\right) .
$$

d. Remarks :

$T$ in the above expressions is the total test time which is defined in Sec. I.A. The above estimators are appropriate for item-censored 1 ife testing either with or without replacement and for time-truncated testing with replacement.

e. Examp1e :

Consider Data Set 1 in Appendix A. Suppose we desire a posterior analysis of the pump failure rate in PWRs (according to the failure to run normal mode) based on the 1972 failure data reported. From past experience suppose that we assign the values 0.50 and $6.0 \times 10^{-6} \mathrm{f} / \mathrm{h}$ to $\alpha_{0}$ and $\beta_{0}$, respectively. In 1972 , six pump failures occurred during a total of approximately 3504000 operating hours for the eight PWRs. The testing situation is considered to be time-truncated 1 ifetesting with replacement. Thus $r=6$ and $T=400(8760)=3504000$. The posterior estimated failure rate is 


$$
\tilde{\lambda}_{6}=\frac{6.0 \times 10^{-6}(6+0.5)}{6.0 \times 10^{-6}(3504000)+1}=1.8 \times 10^{-6} \mathrm{f} / \mathrm{h},
$$

and an upper 95: probability-interval estimate of the failure rate is

$$
\begin{aligned}
\lambda<\left(6.0 \times 10^{-6}\right) & (22.362) /\left[2\left(6.0 \times 10^{-6}\right)(3504000)+2\right] \\
& =3.0 \times 10^{-6} \mathrm{f} / \mathrm{h} .
\end{aligned}
$$

f. General reference:

(i) N. R. Mann, R. E. Schafer, and N. D.

Singpurwalla, Methods for Statistical Analysis of Reliability and Life Data (John Wiley and Sons, New York, 1974). 
III.B.7. Variables test data: empirical Bayes - reliability estimation - - estimated gamma parameters

a. Point estimators of $R(t)$ :

$$
\begin{aligned}
\tilde{R}_{E}(t) & =\left(1+\hat{\beta}_{0} t\right)^{-\hat{\alpha}_{0}} \quad \text { (prior analysis) } \\
& =\left(\begin{array}{l}
\hat{\beta}_{0} T+1 \\
\hat{\beta}_{0} T+\hat{\beta}_{0} t+1
\end{array}\right)^{r+\hat{\alpha}_{0}}, \text { (posterior analysis) }
\end{aligned}
$$

where

$$
\hat{\alpha}_{0}=N \bar{\lambda} /\left(N_{1} \hat{\beta}_{0}\right)
$$

and

$$
\hat{\beta}_{0}=\left(N_{1}^{2} m^{2}-N_{2} N \bar{\lambda}^{2}\right) /\left(N_{1} N_{2} \bar{\lambda}\right)
$$

In these expressions

$$
\begin{gathered}
N_{1}=\sum\left(\frac{r_{j}}{r_{j}-1}\right) ; N_{2}=\sum\left[\frac{r_{j}^{2}}{\left(r_{j}-1\right)\left(r_{j}-2\right)} ;\right] \bar{\lambda}=\sum \hat{\lambda}_{j} / N ; \\
m^{2}=\sum \hat{\lambda}_{j}^{2} / N ; \hat{\lambda}_{j}=r_{j} / T_{j} .
\end{gathered}
$$

If $r_{1}=r_{2} \cdots=r_{N}=r$, these expressions simplify to become

$$
\begin{aligned}
& \hat{\alpha}_{0}=\frac{(r-1) \lambda^{2}}{(r-2) m^{2}-(r-1) \lambda^{-2}} \\
& \hat{\beta}_{0}=\frac{(r-2) m^{2}-(r-1) \bar{\lambda}^{2}}{r \bar{\lambda}} .
\end{aligned}
$$

b. Two-sided probability-interval estimators:

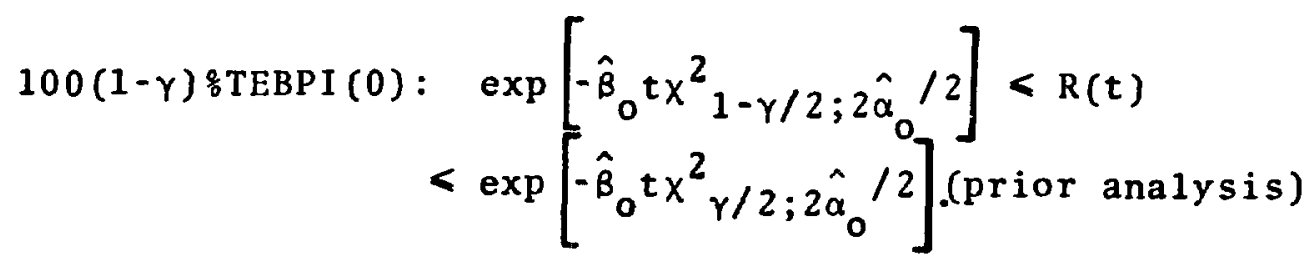




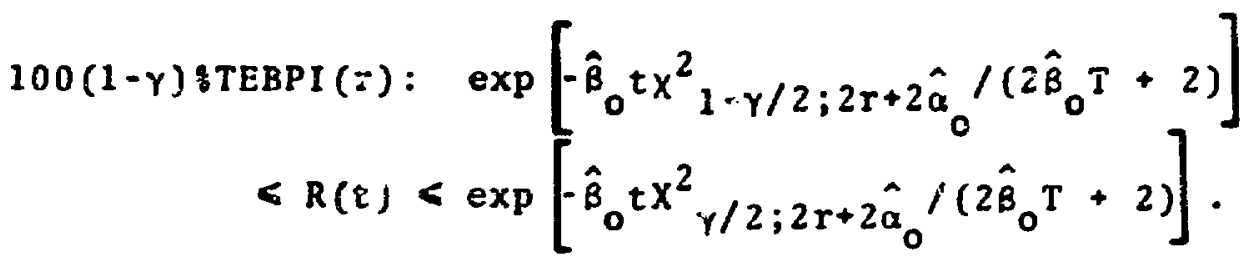

(posterior analysis)

c. Lower one-sided probability-interval estimators:

$100(1-\gamma) \& \operatorname{LEBPI}(0): R(t)>\exp \left[-\hat{\beta}_{0} t \chi^{2} 1-\gamma ; 2 \hat{\alpha}_{0} / 2\right]$.

(prior analysis)

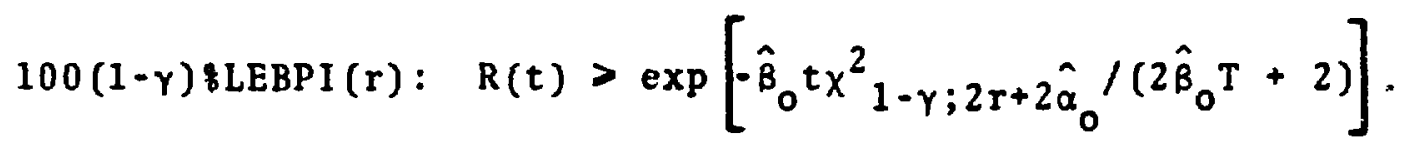

(posterior analysis)

d. Remarks:

$T$ in the above expressions is the total test time which is defined in Sec. I.A. The above estimators are appropriate for item-censored life testing either with or without replacement and for time-truncated testing with repiacement.

The results in $a, b$, and $c$ assume a gamma prior model on $\lambda$ but with unknown parameters $\alpha_{0}$ and $\beta_{0}$. These parameters are estimated from a set of independent test results on the same or similar items. It is assumed that $r_{j}$ failures are observed in total test $T_{j}$ in the $j$ th experiment $(j=1,2, \cdots, N)$. Thus there exists a set of independent failure-rate estimates $\hat{\lambda}_{1}, \hat{\lambda}_{2}, \cdots, \hat{\lambda}_{\mathrm{N}}$ of the item under consideration, where $\hat{\lambda}_{j}=r_{j} / T_{j}$. In a above, all summations range from $j=1$ to $j=N$. It is further required that $r_{j}>2$ in each related experiment. 
-. Example:

Let us use the set of 13 pump failure-rate estimates in Data Set 2 (Appendix B) for estimating $a_{0}$ and $B_{0}$. Unfortunately, the number of failures on which each of the failure-rate estimates is based is unknown. It will be arbitrarily assumed that each estimate is based un 10 failures. Thus $r_{j}=r=10, j=1,2, \cdots, 13$. Now $\lambda=15.24 \times 10^{-6}$ and $\mathrm{m}^{2}=1543.23 \times 10^{-12}$. Thus

$$
\begin{gathered}
\hat{\alpha}_{0}=\frac{(10-1)\left(15.24 \times 10^{-6}\right)^{2}}{(10-2)\left(1543.23 \times 10^{-12}\right)-(10-1)\left(15.24 \times 10^{-6}\right)^{2}}=0.20 \\
\hat{\beta}_{0}=\frac{(10-2)\left(1543.23 \times 10^{-12}\right)-(10-1)\left(15.24 \times 10^{-6}\right)^{2}}{10\left(15.24 \times 10^{-6}\right)}
\end{gathered}
$$$$
=67.29 \times 10^{-6} \mathrm{f} / \mathrm{h} \text {. }
$$

Suppose we now desire a posterior analysis of the annual pump reliability of the PWRs based on the 1972 failure data reported in Data Set 1 (Appendix A). The testing situation is considered to be time-truncated testing with replacement. Thus $T=6$ and $T=400(8760)$

- 3504000 . The posterior estimated annual pump reliability is

$\tilde{R}_{E}(8760)=$

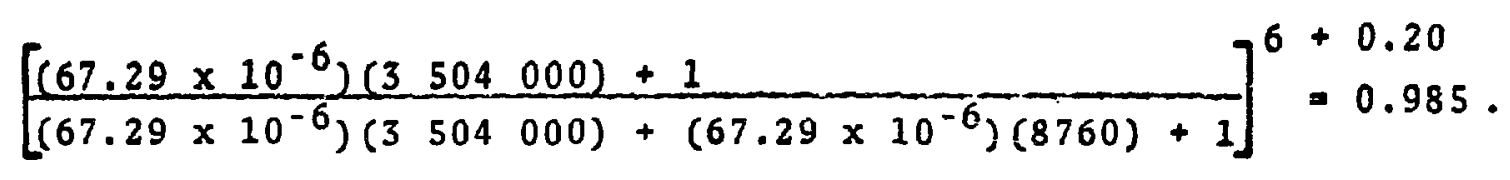

A two-sided $95 \%$ probability-interval estimate of the rellability is 


$$
\begin{gathered}
\exp \left\{-\left(67.29 \times 10^{-6}\right)(8760)(23.337) /\right. \\
\left.\left[(2)\left(67.29 \times 10^{-6}\right)(3504000)+2\right]\right\} \\
<R(8760)<\exp \left\{-\left(67.29 \times 10^{-6}\right)(8760)(4.404) /\right. \\
\left.\left[(2)\left(67.29 \times 10^{-6}\right)(3504000)+2\right]\right\},
\end{gathered}
$$

or

$$
0.97<R(8760)<0.99 .
$$

Note: Since $2 r+\hat{2} \hat{\alpha}_{0}=12.4$, we approximate $x^{2} 0.975 ; 12.4$ $\approx x_{0.975 ; 12}^{2}=23.337$. Similarly, $x_{0.025 ; 12.4}^{2}$ $x_{0.025 ; 12}^{2}=4.404$. Thus, the above interval estimate is only an approximate $95 \%$ probability-interval estimate.

f. General reference:

(i) N. R. Mann, R. E. Schafer, and N. D.

Singpurwa1la, Methods for Statistical Analysis of Reliability and Life Data (John Wiley and Sons, New York, 1974). 
III.B.8. Variables test data: empirical Bayes - reliability estimation - - estimated (empirical) prior

a. Point estimators of $R(t)$ :

$$
\begin{aligned}
\tilde{R}_{E}(\tau) & =\exp \left(-t \sum_{j=1}^{N} \hat{\lambda}_{j} / N\right) \quad \text { (prior analysis) } \\
& =\frac{\sum_{j=1}^{N} \hat{\lambda}_{j} \exp \left[-\hat{\lambda}_{j}(t+T)\right]}{\sum_{j=1}^{N} \hat{\lambda}_{j} \exp \left[-\hat{\lambda}_{j} T\right]} \quad \text { (posterior analysis) }
\end{aligned}
$$

b. Two-sided probability-interval estimators:

(see Reference (iii) below)

c. Lower one-sided probability-interval estimators: (see Reference (iii) below)

d. Remarks:

$T$ in the expression in $a$ is the total test ime which is defined in Sec. I.A. The above estimators are appropriate for item-censored 1 ife testing either with or without replacement and for time-truncated testing with replacement.

The results in a above do not require an assumption regarding a type of prior model, such as a gamma model. Rather, the complete prior distribution is empirically estimated from a set of independent test results on the same or similar items. It is assumed that $r_{j}$ failures are observed in total test time $T_{j}$ in the $j$ th experiment $(j=1,2, \cdots, N)$. Thus there exists a set of independent failure-rate estimates $\hat{\lambda}_{1}, \hat{\lambda}_{2}, \cdots, \hat{\lambda}_{N}$ of the item under consideration, where $\hat{\lambda}_{j}=r_{j} / T_{j}$. 


\section{e. Example:}

Let us use the set of 13 pump failure-rate estimates in Data Set 2 (Appendix B) to form a posterior estimate of the annual pump reliability in PWRs based on the 1972 failure data reported in Data Set 1 (Appendix A). The testing situation is considered to be timetruncated testing with replacement. Thus $r=6$ and $T$ $=400(8760)=3,504,000$. The posterior estimated annual pump reliability is

$\tilde{R}_{E}(8760)=\frac{\left(1.3 \times 10^{-5}\right)^{6} \exp \left[-\left(1.3 \times 10^{-5}\right)(8760+3504\right.}{\left(1.3 \times 10^{-5}\right)^{6} \exp \left[-\left(1.3 \times 10^{-5}\right)(3504000)\right]}$

$\frac{+\left(3.0 \times 10^{-6}\right)^{6} \exp \left[-\left(3.0 \times 10^{-6}\right)(8760+3504000)\right]}{+\left(3.0 \times 10^{-6}\right)^{6} \exp \left[-\left(3.0 \times 10^{-6}\right)(3504000)\right]}$

$$
\begin{aligned}
& \left.\frac{+\cdots+(4.0}{+\cdots+\left(4.0 \times 10^{-6}\right)}\right)_{6}^{6} \exp \left[-\left(4.0 \times 10^{-6}\right)(8760 * 3504000)\right] \\
& \quad=0.98 .
\end{aligned}
$$

f. General references:

(i) G. H. Lemon, "An Empirical Bayes Approach to Reliabiiity," IEEE Trans. Re1.R-21, 155-188 (August 1972).

(ii) H. F. Martz, Jr., "Pooling Life-Test Data by Means of the Empirical Bayes Method," IEEE Trans. Re1. R-24, 27-30 (April 1975).

(iii) M. G. Lian, "Bayes and Empirical Bayes Estimation of Reliability for the Weibull Model," unpublished Ph.D. dissertation, Texas Tech University, University Microfilms, Ann Arbor, Michigan (1975). 
III.B.9. Variables test data: empirical Bayes - failure-rate estimation - - estimated gamma parameters

a. Point estinators of $\lambda$ :

$$
\begin{aligned}
\tilde{\lambda}_{E} & =\hat{\alpha}_{0} \hat{\beta}_{0}, & & \text { (prior analysis) } \\
& =\frac{\hat{\beta}_{0}\left(r+\hat{\alpha}_{0}\right)}{\hat{\beta}_{0} T+1}, & & \text { (posterior analysion }
\end{aligned}
$$

where

$$
\hat{\alpha}_{0}=N \bar{\lambda} /\left(N_{1} \hat{\beta}_{0}\right) \text {, }
$$

and

$$
\hat{\beta}_{0}=\left(N_{1}^{2} m^{2}-N_{2} N \bar{\lambda}^{2}\right) /\left(N_{1} N_{2} \bar{\lambda}\right) .
$$

In these expressions

$$
\begin{aligned}
& N_{1}=\sum\left(\frac{r_{j}}{r_{j}-1}\right) ; N_{2}=\sum\left[\frac{r_{j}^{2}}{\left(r_{j}-1\right)\left(r_{j}-2\right)}\right] ; i=\sum \hat{\lambda}_{j} / N ; \\
& m^{2}=\sum \hat{\lambda}_{j}^{2} / N ; \hat{\lambda}_{j}=r_{j} / T_{j} .
\end{aligned}
$$

If $r_{1}=r_{2}=\cdots=r_{N}=r$, these expressions simplify to become

$$
\begin{aligned}
& \hat{\alpha}_{0}=\frac{(r-1) \bar{\lambda}^{2}}{(r-2) m^{2}-(r-1) \bar{\lambda}^{-2}}, \\
& \hat{\beta}_{0}=\frac{(r-2) m^{2}-(r-1) \bar{\lambda}^{2}}{r \bar{\lambda}} .
\end{aligned}
$$


b. Two-sided probability-interval estimators:

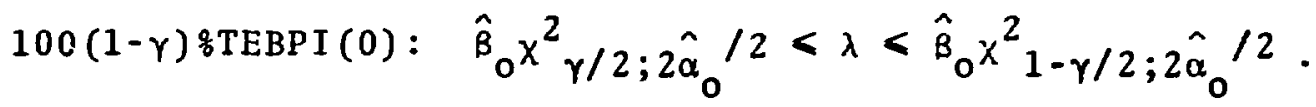

(prior analysis)

$\begin{aligned} 100(1-\gamma) \& \operatorname{TEBPI}(r): & \hat{\beta}_{0} x^{2} / 2 ; 2 \mathrm{r}+2 \hat{\alpha}_{0} /\left(2 \hat{\beta}_{0} T+2\right)<\lambda \\ < & \hat{\beta}_{0} x^{2} 1-\gamma / 2 ; 2 r+2 \hat{\alpha}_{0} /\left(2 \hat{\beta}_{0} T+2\right) .\end{aligned}$

(posterior analysis)

c. Upper one-sided probability-interval estimators:

$100(1-\gamma) \& \operatorname{UEBPI}(0): \quad \lambda<\hat{\beta}_{0} \chi_{1-\gamma ; 2 \hat{\alpha}_{0}^{2}}^{2} . \quad$ (prior analysis)

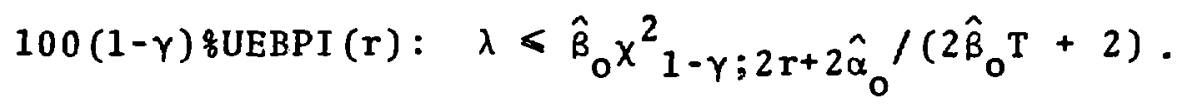

(posterior analysis)

d. Remarks:

$T$ in the above expressions is the total test time which is defined in Sec. I.A. The above estimators are appropriate for item-censored life testing either with or without replacement and for time-truncated testing with replacement.

The results in $a, b$, and $c$ assume a gamma prior model on $\lambda$ but with unknown parameters $\alpha_{0}$ and $\beta_{0}$. These parameters are estimated from a set of independent test results on the same or similar items. It is assumed that $r_{j}$ failures are observed in total test time $T_{j}$ in the ${ }_{j}$ th experiment $(j=1,2, \cdots, N)$. Thus there exists a set of independent failure-rate estimates $\hat{\lambda}_{1}, \hat{\lambda}_{2}, \cdots, \hat{\lambda}_{N}$ of the item under consideration, where $\hat{\lambda}_{j}$ $=\mathbf{r}_{j} / \mathrm{T}_{j}$. In a above, all summations range from $j=1$ 
to $j=N$. It is further required that $r_{j}>2$ in each related experiment.

e. Example:

Let us use the set of 13 pump failure-rate estimates in Data Set 2 (Appendix B) for estimating $\alpha_{0}$ and $\beta_{0}$. Unfortunately, the number of failures used to compute each failure-rate estimate is unknown. It will be arbitrarily assumed that each estimate is based upon 10 failures. Thus $r_{j}=r=10, j=1,2, \cdots, 13$. Now $\bar{\lambda}=15.24 \times 10^{-6}$ and $m^{2}=1543.23 \times 10^{-12}$. Thus $\hat{\alpha}_{0}=\frac{(10-1)\left(15.24 \times 10^{-6}\right)^{2}}{(10-2)\left(1543.23 \times 10^{-12}\right)-(10-1)\left(15.24 \times 10^{-6}\right)^{2}}=0.20$, $\hat{\beta}_{0}=\frac{(10-2)\left(1543.23 \times 10^{-12}\right)-(10-1)\left(15.24 \times 10^{-6}\right)^{2}}{10\left(15.24 \times 10^{-6}\right)}$, $=67.29 \times 10^{-6} \mathrm{f} / \mathrm{h}$.

Suppose we ncw desire a posterior estimate of the pump failure rate, for the failure to run normal mode, based on the 1972 failure data reported in Data Set 1 (Appendix A). The testing situation is considered to be timetruncated testing with replacement. Thus $r=6$ and $T=400(8760)=3504000$. The posterior estimated failure-rate is

$\tilde{\lambda}_{E}=\frac{67.29 \times 10^{-6}(6+0.20)}{67.29 \times 10^{-6}(3504000)+1}=1.8 \times 10^{-6} \mathrm{f} / \mathrm{h}$.

and a two-sided 95\% probability-interval estimate of the failure-rate is

$\left(67.29 \times 10^{-6}\right)(4.404) /\left[(2)\left(67.29 \times 10^{-6}\right)(3504000)+2\right]$ 
$<\lambda<\left(67.29 \times 10^{-6}\right)(23.337) /\left[(2)\left(67.29 \times 10^{-6}\right)(3504000)+2\right]$,

or

$6.3 \times 10^{-7} \mathrm{f} / \mathrm{h}<\lambda<3.3 \times 10^{-6} \mathrm{f} / \mathrm{h}$.

f. General reference:

(i) N. R. Mann, R. E. Schafer, and N. D.

Singpurwalla, Methods for Statistical Analysis of $\backslash$ Re-

liability and Life Data (John Wiley and Sons, New York, 1974). 
III.B.10. Variables test data: empirical Bayes - failure-rate estimation - estimated (empirical) prior

a. Point estimators of $\lambda$ :

$$
\begin{array}{rlr}
\tilde{\lambda}_{E} & =\sum_{j=1}^{N} \hat{\lambda}_{j} / N, & \text { (prior analysis) } \\
& =\frac{\sum_{j=1}^{N} \hat{\lambda}_{j}^{r+1} \exp \left(-\hat{\lambda}_{j} T\right)}{\sum_{j=1}^{N} \hat{\lambda}_{j}^{r} \exp \left(-\hat{\lambda}_{j} T\right)} .
\end{array}
$$

b. Two-sided probability-interval estimators:

(see Reference (iii) below).

c. Upper one-sided probability-interval estimators:

(see Reference (iii) below).

d. Remarks:

$T$ in the expression in $a$ is the total test time which is defined in Sec. I.A. The above estimators are appropriate for item-censored 1 ife testing either with or without replacement and for time-truncated testing with replacement.

The results in a above do not require an assumption regarding a type of prior model, such as a gamma model. Rather, the complete prior distribution is empirically estimated from a set of independent failure-rate estimates $\hat{\lambda}_{1}, \hat{\lambda}_{2}, \cdots, \hat{\lambda}_{\mathrm{N}}$ or the item under consideration, where $\hat{\lambda}_{j}=r_{j} / T_{j}$. 
e. Example:

Let us use the set of 13 pump failure-rate estimates in Data Set 2 (Appendix B) to form a posterior estimate of the pump failure rate in PWRs based on the 1972 failure data reported in Data Set 1 (Appendix A). The testing situation is considered to be timetruncated testing with replacement. Thus $r=6$ and $T=400(8760)=3504000$. The posterior estimated pump failure-rate is

$$
\begin{aligned}
\tilde{\lambda}_{E}= & \frac{\left(1.3 \times 10^{-5}\right)^{7} \exp \left[-\left(1.3 \times 10^{-5}\right)(3504000)\right]}{\left(1.3 \times 10^{-5}\right)^{6} \exp \left[-\left(1.3 \times 10^{-5}\right)(3504000)\right]} \\
& +\left(3.0 \times 10^{-6}\right)^{7} \exp \left[-\left(3.0 \times 10^{-6}\right)(3504000)\right] \\
& +\left(3.0 \times 10^{-6}\right)^{6} \exp \left[-\left(3.0 \times 10^{-6}\right)(3504000)\right] \\
& +\cdots+\left(4.0 \times 10^{-6}\right)^{7} \exp \left[-\left(4.0 \times 10^{-6}\right)(3504000)\right] \\
& +\ldots+\left(4.0 \times 10^{-6}\right)^{6} \exp \left[-\left(4.0 \times 10^{-6}\right)(3504000)\right] \\
= & 2.3 \times 10^{-6} \mathrm{f} / \mathrm{h} .
\end{aligned}
$$

f. General references:

(i) G. H. Lemon, "An Empirical Bayes Approach to Reliability," IEEE Trans. Rel.R-21, 155-158 (August 1972).

(ii) H. F. Martz, Jr., "Pooling Life-Test Data by Means of the Empirical Bayes Method," IEEE Trans. Re1 R-24, 27-30 (Apri1 1975).

(iii) M. G. Lian, "Bayes and Empirical Bayes Estimation of Reliability for the Weibull Model, unpublished Ph.D. dissertation, Texas Tech University, University Microfilms, Ann Arbor, Michigan (1975). 


\section{REFERENCES}

1. R. A. Waller and H. F. Martz, Jr., "Bayesian Reliability Estimation: The State of the Art for the Time-Dependent Case." Los Alamos Scientific Laboratory report LA-6003 (October 1975).

2. H. F. Martz, Jr., and R. A. Waller, "The Basies of Bayesian Reliability Estimation from Attribute Test Data," Los Alamos Scientific Laboratory report LA-6126 (February 1976).

3. M. S. Waterman, H. F. Martz, and R. A. Waller, "Fitting Beta Prior Distributions in Bayesian Reliability Analysis," Los Alamos Scientific Laboratory report LA-6395-MS (June 1976).

4. H. F. Martz and M. Horita, "Fitting Gamma Prior Distributions in Bayesian Reliability Analysis," under preparation.

5. R. A. Waller, H. F. Martz, M. Horita, and M. S. Waterman, "Fitting Gamma Prior Distributions in Bayesian Reliability Analysis Based on Reliability Considerations," under preparation. 


\section{APPENDICES}

A. Example Data Set 1

B. Example Data Set 2

C. Percentage Points of the F-Distrioution

D. Percentiles of the $x^{2}$ - Distribution 


\section{A. DENDIX A}

\section{Example Data Set 1}

The following data are taken from Appendix III (FailureData) of WASH-1400 "Reactor Safety Study", U. S. Nuclear Regulatory Commission, October 1975, pp. III 35-36. The data consist of the number of pump failures observed in 1972 in eight pressurized water reactors (PWR) in commercial operation in the $U . S$. The designated failure mode was failure to run normal.

\begin{tabular}{lllllll}
\hline $\mathbf{i}$ & \multicolumn{1}{c}{ PWR } & $\mathrm{n}_{\mathrm{i}}$ & $\mathrm{r}_{\mathbf{i}}$ & $\mathrm{x}_{\mathbf{i}}$ & $\mathrm{T}(\mathrm{h})$ & $\hat{\mathrm{R}}_{\mathbf{i}}$ \\
\hline 1 & Haddam Neck & 50 & 0 & 50 & 438000 & 1.00 \\
2 & Yankee Rowe & 50 & 0 & 50 & 438000 & 1.00 \\
3 & Indian Point 1 & 50 & 1 & 49 & 438000 & 0.98 \\
4 & San Onofre 1 & 50 & 1 & 49 & 438000 & 0.98 \\
5 & Ginna & 50 & 0 & 50 & 438000 & 1.00 \\
6 & Point Beach 1 & 50 & 0 & 50 & 438000 & 1.00 \\
7 & Robinson 2 & 50 & 1 & 49 & 438000 & 0.98 \\
8 & Palisades & 50 & 3 & 47 & 438,000 & 0.94 \\
\hline
\end{tabular}

Here $n_{i}$ denotes the number of pumps in service and $r_{i}$ represents the number of observed pump failures. Also, $x_{i}$ denotes the number of survivors, $T$ is the total test time for 1972 , and $\hat{R}_{i}=$ $x_{i} / n_{i}$ 
The following data are taken from Apfiendix III (Failure Data) of WASH-1400 "Reactor Safety Study", U. S. Nuciear Regulatory Commission, October 1972, pp. III 7-8. The data consist of failure-rate estimates of pumps for a failure to run normal mode from several published sources.

\begin{tabular}{|c|c|}
\hline SOURCE* & FAILURE-RATE $(f / h)$ \\
\hline AVCO & $1.3 \times 10^{-5}$ \\
\hline FARADA & $3.0 \times 10^{-6}$ \\
\hline LMEC & $1.4 \times 10^{-4}$ \\
\hline SRS & $1.0 \times 10^{-5}$ \\
\hline HOLMES HN-190 & $3.0 \times 10^{-6}$ \\
\hline SHOP US-NUC & $1.4 \times 10^{-7}$ \\
\hline BOURNE UK & $2.0 \times 10^{-6}$ \\
\hline UNDERAKES (GERMAN) & $1.0 \times 10^{-5}$ \\
\hline DAVIL & $3.0 \times 10^{-6}$ \\
\hline EUROPE NUC AGENCY & $1.0 \times 10^{-6}$ \\
\hline PUGH & $3.0 \times 10^{-6}$ \\
\hline OTWAY & $6.0 \times 10^{-6}$ \\
\hline PROCEEDINGS & $4.0 \times 10^{-6}$ \\
\hline
\end{tabular}

*Complete References Given in WASH-1400. 
Here $\bar{\lambda}$ and $S$ are the mean and standard deviation, respectively, of the 13 failure-rate estimates. Also, WASH-1400 gives the median failure-rate estimate as $3.0 \times 10^{-5}$ and lower and upper bounds as $3 \times 10^{-6}$ and $3 \times 10^{-4}$, respectively. These estimates are based on a $10 \mathrm{~g}$-normal distribution and coincide with the approximate $5 \%$ and $95 \%$ range end points. The interval is thus an approximate $90 \%$ probability interval. 
- ดี

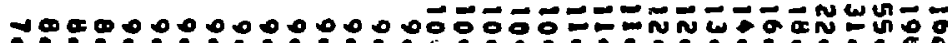
$\div 0$

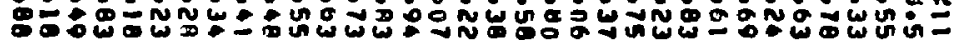

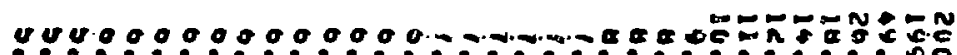

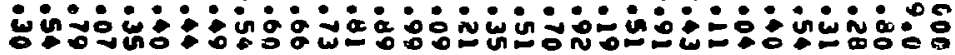

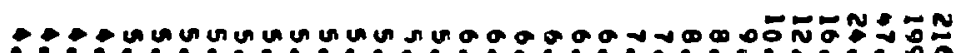

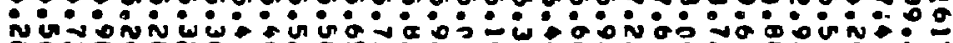

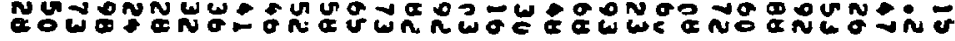

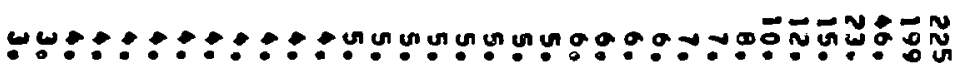

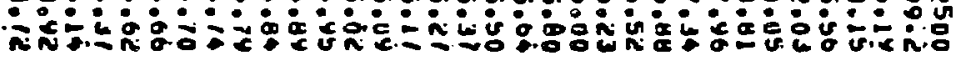

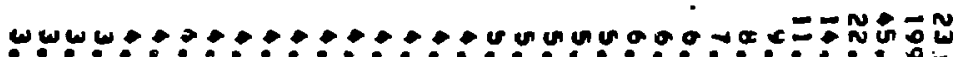

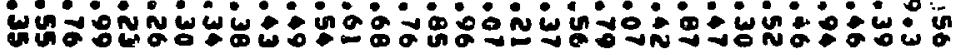

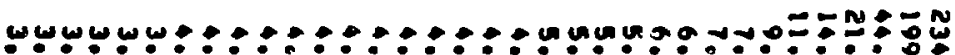

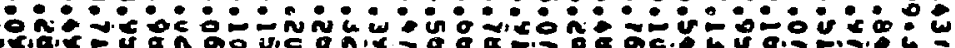

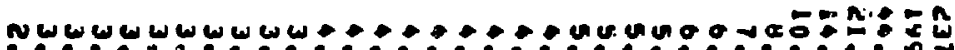

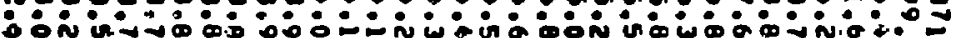

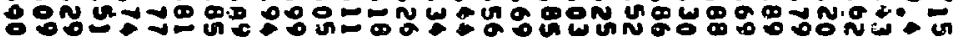

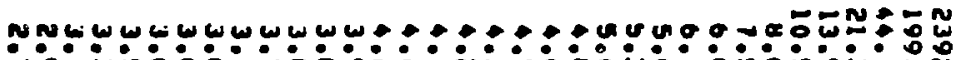

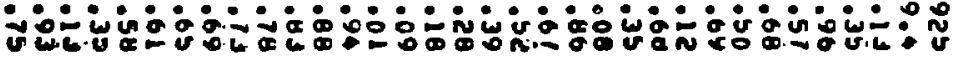

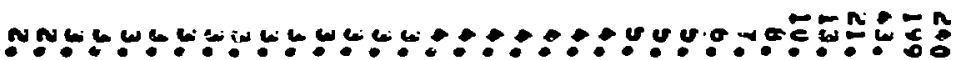

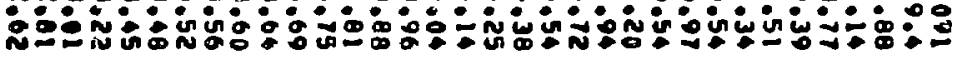

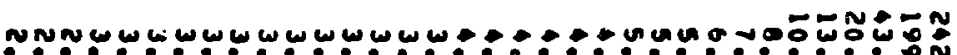
- : - : -

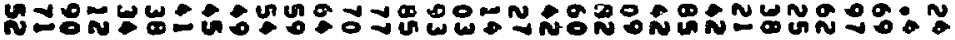
N NNN

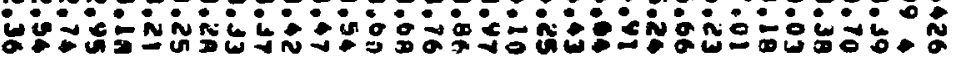

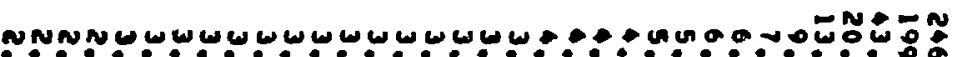

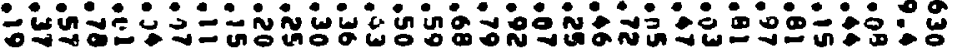
NONAN YN UN W - $\cdot-0$

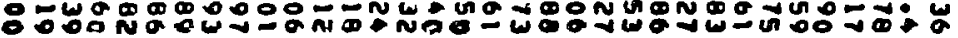

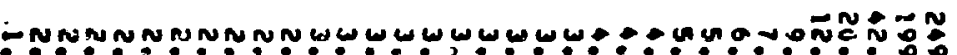

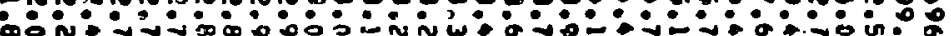

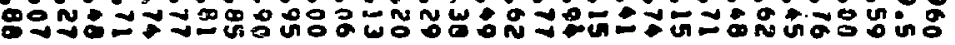

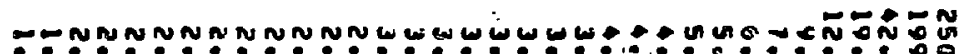

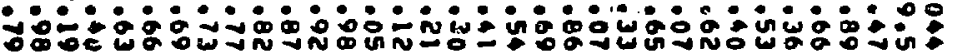

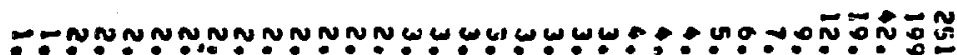

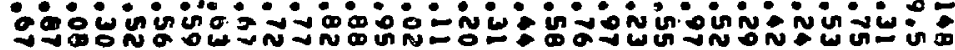

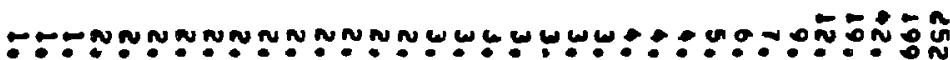

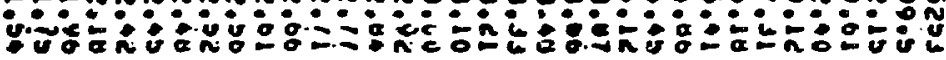
־ะก

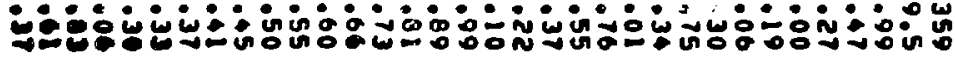
○一ำ

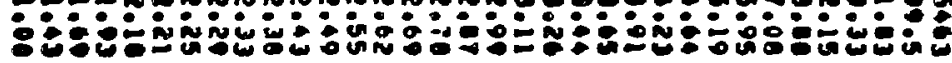




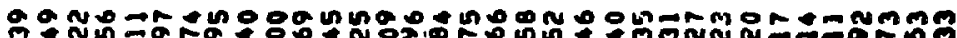
m:Nน ร马:

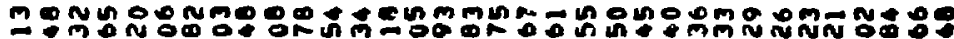
今0:

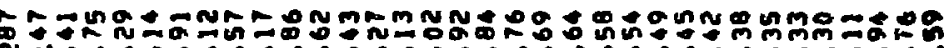

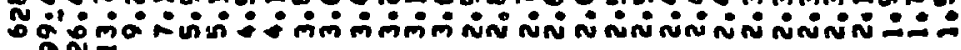

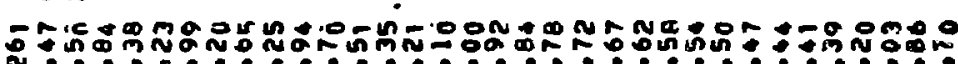

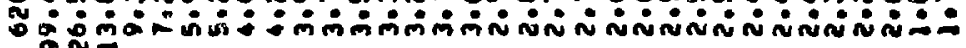

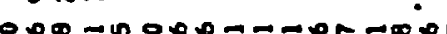

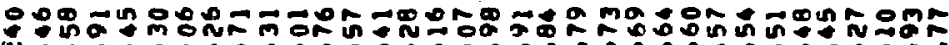

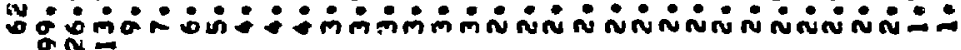
$0 \mathrm{n}=$

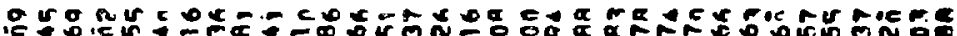

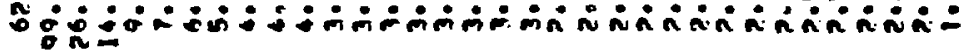

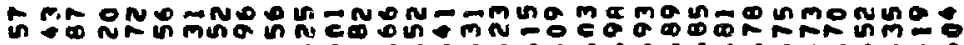

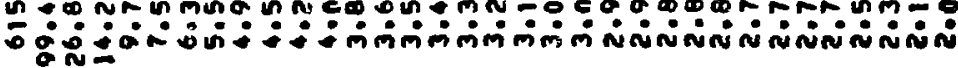

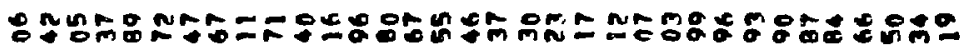

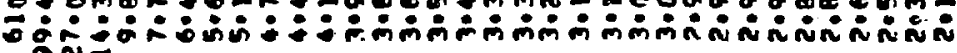

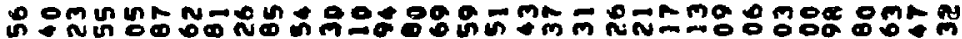
"gñ

(1)

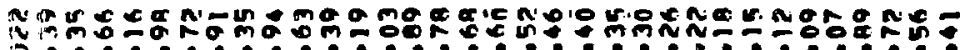

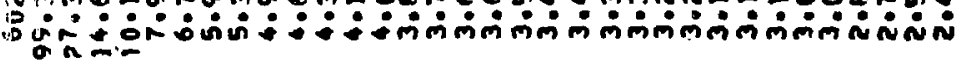

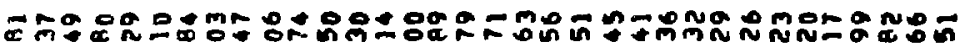

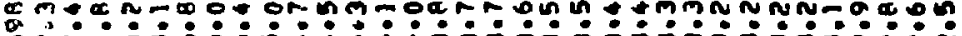

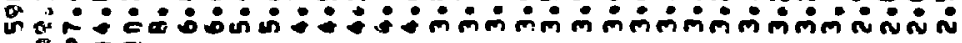

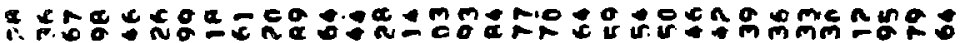

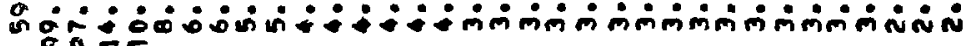

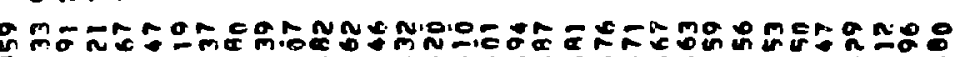
ân

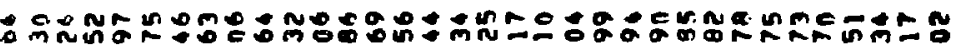
ن

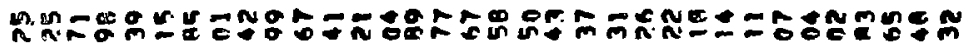

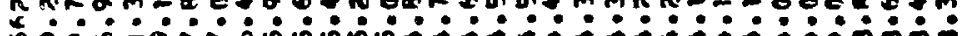

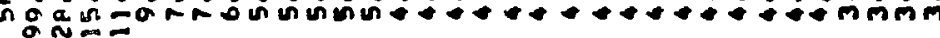

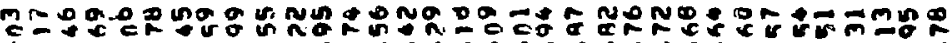

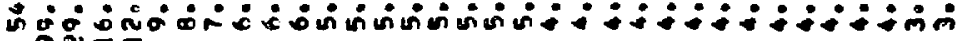

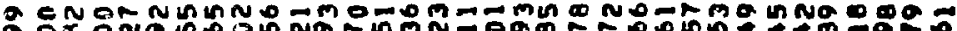
\%

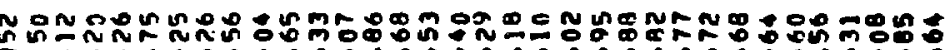

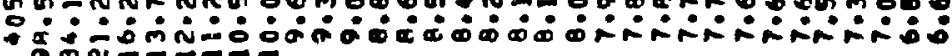

$\mid$




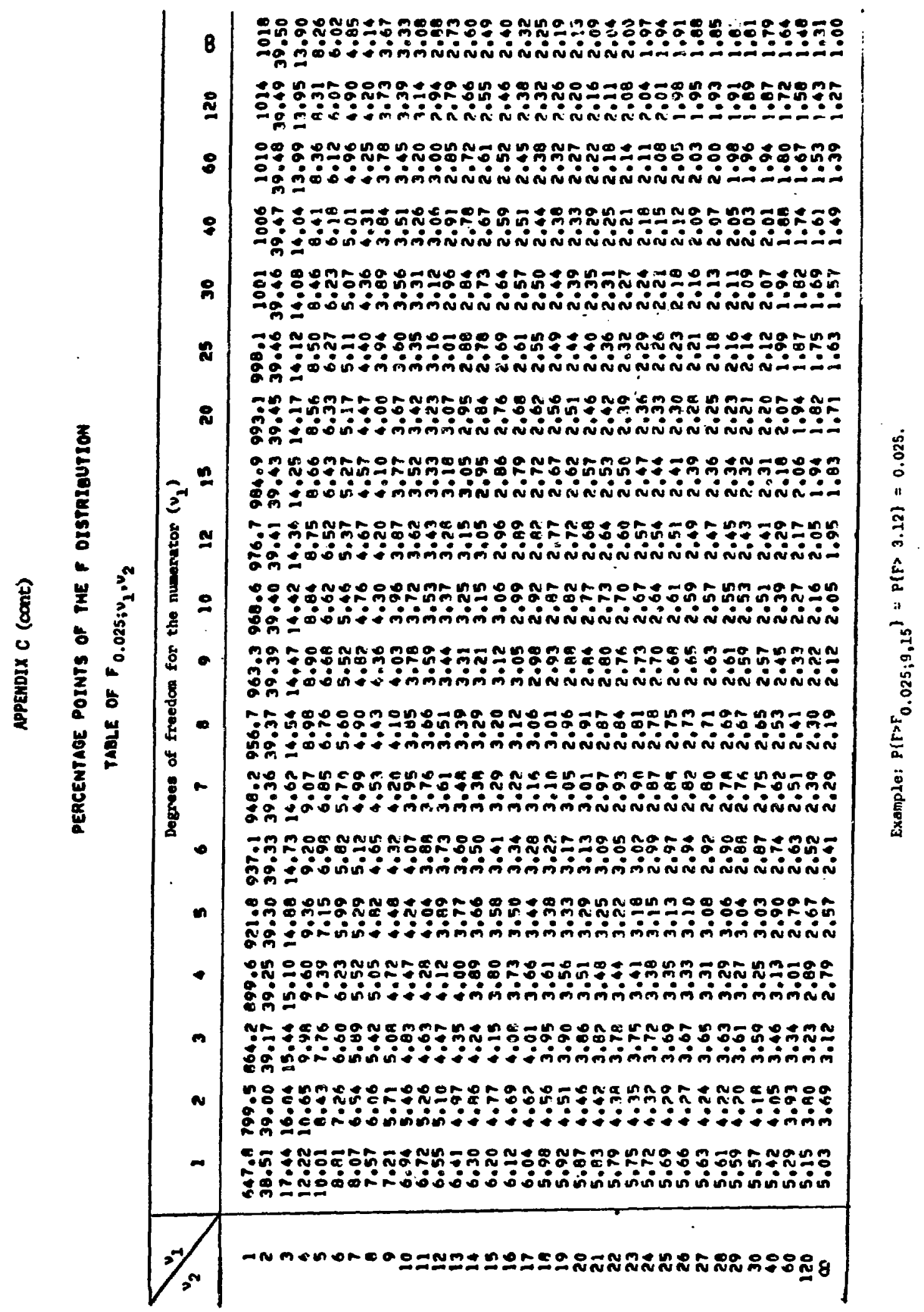

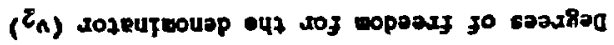




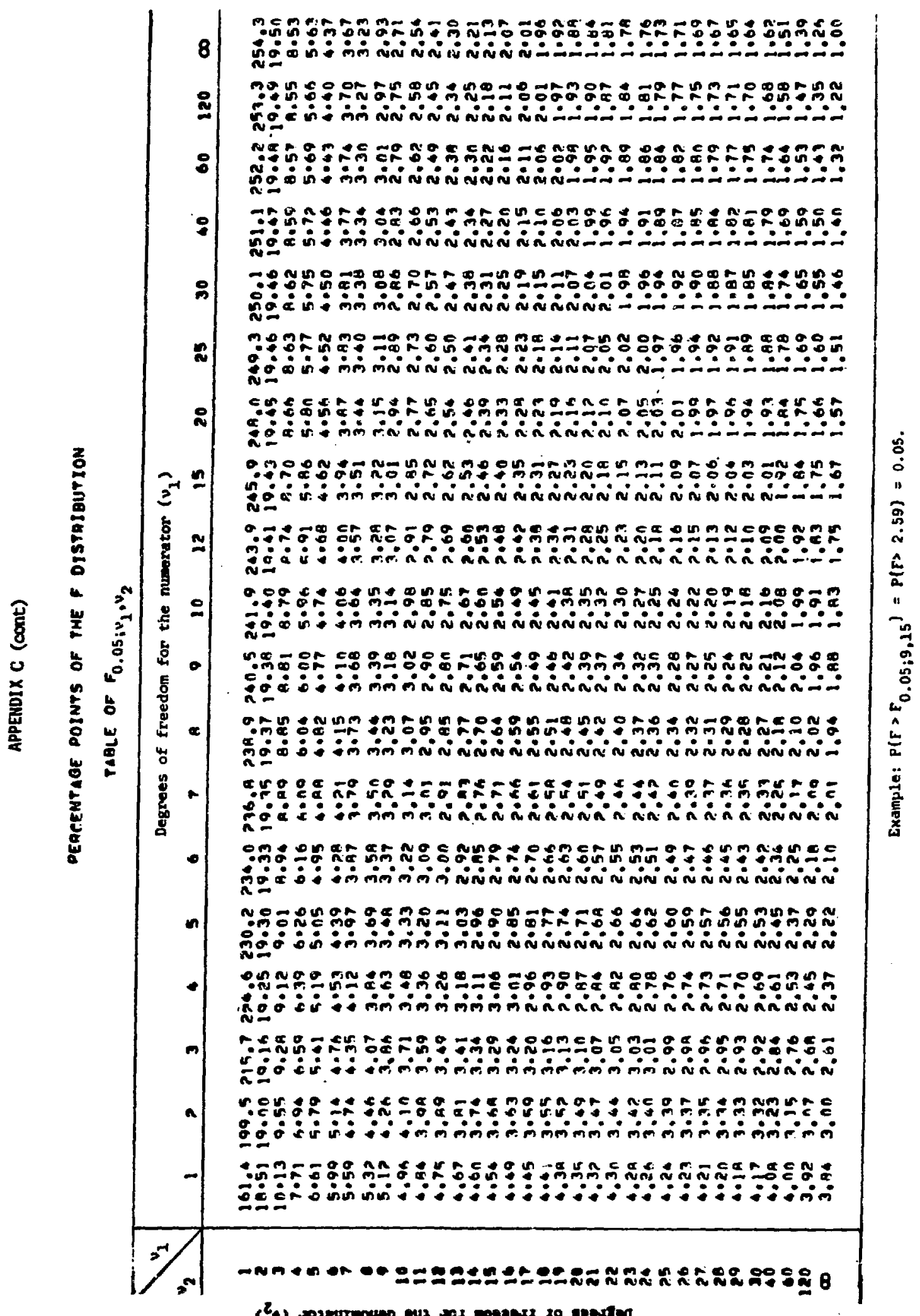

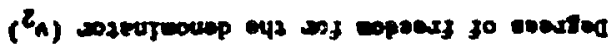




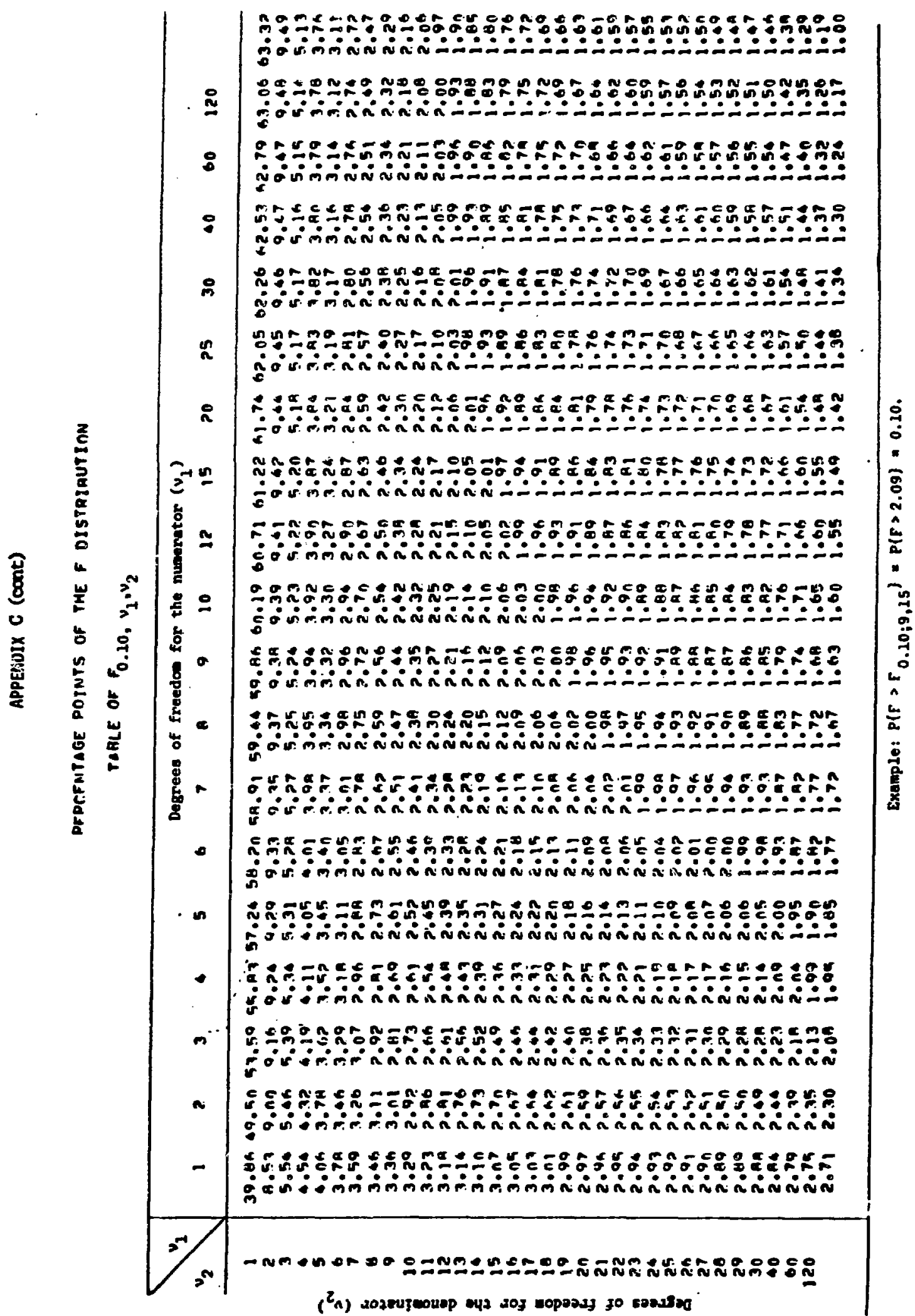




\section{APPENDIX D}

Percentiles of the $x^{2}$-Distribution ${ }^{a}$

$$
\text { Table of } x_{\alpha ; \nu}^{2}
$$

\begin{tabular}{|c|c|c|c|c|c|c|}
\hline$v 0^{\alpha}$ & 0.005 & 0.025 & 0.050 & 0.950 & 0.975 & 0.995 \\
\hline 1 & 0.000 & 0.001 & 0.004 & 3.841 & 5.024 & 7.879 \\
\hline 2 & 0.010 & 0.051 & 0.103 & 6.991 & 7.378 & 10.597 . \\
\hline 3 & 0.072 & 0.216 & 0.352 & 7.815 & 9.348 & 12.838 \\
\hline 4 & 0.207 & 0.484 & $0.7 \mathrm{i} 1$ & 9.488 & 11.143 & 14.860 \\
\hline 5 & 0.412 & 0.831 & 1.145 & 11.070 & 12.833 & 16.750 \\
\hline 6 & 0.676 & 1.237 & 1.635 & 12.592 & 14.449 & 18.548 \\
\hline 7 & 0.989 & 1.690 & 2.167 & 14.067 & 16.013 & 20.278 \\
\hline 8 & 1.344 & 2.180 & 2.733 & 15.507 & 17.535 & 21.955 \\
\hline 9 & 1.735 & 2.700 & 3.325 & 16.919 & 19.023 & 23.589 \\
\hline 10 & 2.156 & 3.247 & 3.940 & 18.307 & 20.483 & 25.188 \\
\hline 11 & 2.603 & 3.816 & 4.575 & 18.675 & 21.920 & 26.757 \\
\hline 12 & 3.074 & 4.404 & 5.226 & 21.026 & 23.337 & 28.300 \\
\hline 13 & 3.565 & 5.009 & 5.892 & 22.362 & 24.736 & 29.819 \\
\hline 14 & 4.075 & 5.629 & 6.671 & 23.685 & 26.119 & 31.319 \\
\hline 15 & 4.601 & 6.262 & 7.261 & 24.996 & 27.488 & 32.801 \\
\hline 16 & 5.142 & 6.908 & 7.962 & 26.296 & 28.845 & 34.267 \\
\hline 17 & 5.697 & 7.564 & 8.672 & 27.587 & 30.191 & 35.718 \\
\hline 18 & 6.265 & 8.231 & $\mathbf{9 . 3 9 0}$ & 28.869 & 31.526 & 37.156 \\
\hline 19 & 6.844 & 8.907 & 10.117 & 30.144 & 32.852 & 38.582 \\
\hline 20 & 7.434 & 9.591 & 10.851 & 31.410 & 34.170 & 39.997 \\
\hline 21 & 8.034 & 10.283 & 11.591 & 32.671 & 35.479 & 41.401 \\
\hline 22 & 8.643 & 10.982 & 12.338 & 33.924 & 36.781 & 42.796 \\
\hline 23 & 9.260 & 11.689 & 13.091 & 35.172 & 38.076 & 44.181 \\
\hline 24 & 9.886 & 12.401 & 13.848 & 36.415 & 39.364 & 45.559 \\
\hline 25 & 10.520 & 13.120 & 14.611 & 37.652 & 40.646 & 46.928 \\
\hline 26 & 11.160 & 13.844 & 15.379 & 38.885 & 41.923 & 48.290 \\
\hline 27 & 11.808 & 14.573 & 16.151 & 40.113 & 43.195 & 49.645 \\
\hline 28 & 12.461 & 15.308 & 16.928 & 41.337 & 44.461 & 50.993 \\
\hline 29 & 13.121 & 16.047 & 17.708 & 42.557 & 45.722 & 52.336 \\
\hline 30 & 13.787 & 16.791 & 18.493 & 43.773 & 46.979 & 53.672 \\
\hline 60 & 35.534 & 40.482 & 43.188 & 79.032 & 83.298 & 91.952 \\
\hline 100 & 67.328 & 74.222 & 77.929 & 124.342 & 129.561 & 140.169 \\
\hline 110 & 75.550 & 82.867 & 86.792 & 135.480 & 140.917 & 151.948 \\
\hline 120 & 83.852 & 91.573 & 95.705 & 146.567 & 152.211 & 163.648 \\
\hline
\end{tabular}

Example: $P\left\{x^{2}<x_{0.95 ; 10}^{2}\right\}=P\left\{x^{2}<18.307\right\}=0.95$ 